\title{
Chromatin: a tunable spring at work inside chromosomes
}

\author{
Eli Ben-Haïm, Annick Lesne and Jean-Marc Victor* \\ Laboratoire de Physique Théorique des Liquides, \\ Université Pierre et Marie Curie, \\ Case courrier 121, 4 Place Jussieu, 75252 Paris Cedex 05, France
}

(October 26, 2018)

\begin{abstract}
This paper focuses on mechanical aspects of chromatin biological functioning. Within a basic geometric modeling of the chromatin assembly, we give for the first time the complete set of elastic constants (twist and bend persistence lengths, stretch modulus and twist-stretch coupling constant) of the so-called 30-nm chromatin fiber, in terms of DNA elastic properties and geometric properties of the fiber assembly. The computation naturally embeds the fiber within a current analytical model known as the "extensible worm-like rope", allowing a straightforward prediction of the forceextension curves. We show that these elastic constants are strongly sensitive to the linker length, up to $1 \mathrm{bp}$, or equivalently to its twist, and might locally reach very low values, yielding a highly flexible and extensible domain in the fiber. In particular, the twist-stretch coupling constant, reflecting the chirality of the chromatin fiber, exhibits steep variations and sign changes when the linker length is varied. We argue that this tunable elasticity might be a key feature for chromatin function, for instance in the initiation and regulation of transcription.
\end{abstract}

PACS numbers: 82.37.Rs, 46.25.-y, 87.19.Rr, 83.80.Lz

*Email adresses for correspondence: lesne@lptl.jussieu.fr, victor@lptl.jussieu.fr 


\section{INTRODUCTION}

Chromatin is an ubiquitous protein-DNA complex observed in chromosomes of all eukaryotic organisms and strikingly conserved during evolution [1] [3]. It ensures DNA compaction during the mitosis and plays a key but still unclear role in all the biological functions involving genomic DNA: replication, transcription and repair. For instance, the chromatin degree of compaction is acknowledged to regulate, somehow, transcriptionally active regions [4].

The general issue taken up in this paper is to understand the interplay between the mechanical properties of the fiber and its biological functions. We aim at understanding quantitatively the grounds for existence of the so universal and so conserved features of the assembly of linkers and nucleosomes forming the chromatin fiber. We claim that part of the answer lays in the peculiar mechanical properties of this assembly.

The typical length scale of chromatin fiber (diameter about $30 \mathrm{~nm}$ ) is large enough to allow a mechanistic viewpoint: describing the elastic properties of the chromatin fiber is nothing but a problem of spring mechanics [5]. Nevertheless, the architecture of this "spring" is much more complex than a simple helical coiling and we expect that the detailed structural features of the chromatin assembly still strongly influence the behavior at the fiber scale. We thus investigate the specific elastic behavior associated with the chromatin structure and its sensitivity to the structural parameters of the fiber, singled out within a basic geometric modeling of its assembly.

We underline that fiber elasticity is involved in two, quite different, issues. A first one is to describe the linear elastic response of the fiber to global stresses, i.e. a force and a torque applied at its ends. This issue refers to micromanipulations in which a single chromatin fiber is pulled (and possibly will be twisted) [6]. Our theoretical study provides a framework to interpret the experimental results, in particular to predict force-extension curves in terms of geometric and mechanical parameters of the underlying model of fiber. Our approach largely extends preliminary results presented quite recently by Schiessel et al. [7], since it gives the exact analytical value of the complete set of elastic constants of the fiber. Comparison with the observed curves allows to extract small scale information, either about the microscopic structure, either about the interactions involved, either about possible conformational or structural changes.

However, except during anaphase, when sister chromatids are separated by the mitotic spindle [8], such "macroscopic" stresses are not encountered in vivo at the chromatin fiber level; micromanipulations of the fiber and associated force measurements may nevertheless unravel physical parameters involved in biological mechanisms, such as the elastic constants. An issue directly relevant to the in vivo functioning of chromatin is to de- scribe the response of the fiber to local, internal stresses as those created by intercalators, groove-binding proteins, or any induced change in the fiber assembly or small-scale structure. Our theoretical approach also gives a framework to such studies. Indeed, to be solved, both issues require to relate the structure and mechanics at the DNA scale and those at fiber scale, which is the scope of the present work.

The chromatin scale is precisely the scale of nanomechanics: at this scale, we expect a strong and direct interplay between the biological functioning, monitored by various enzymes, and the mechanical properties of the substrate, here the chromatin fiber. We thus believe that a mechanistic approach is well-suited to evidence possible mechanisms for the fiber decondensation prior to transcription, for the connection between enhancer and promoter DNA regions during transcription, for the nucleosomal DNA site exposure allowing protein binding at specific sites or for the ejection of nucleosomes presumably required during replication [9].

The core of the study is to describe how the linear elastic behavior of chromatin fiber originates from the elastic properties of linker DNA (i.e. naked DNA connecting the nucleosomes). In Section II, we introduce a mechanical model of chromatin fiber, quite similar to the so-called two-angle model [10]; it incorporates microscopic data such as DNA structure and elastic properties 11] 12 as well as nucleosome cristallographic data 13 into an analytically tractable assembly. Its implementation gives the geometric properties of the chromatin fiber, presented in Section III with a special mention to symmetry properties and to the quantities relevant to the mechanics of the fiber. Section IV is devoted to the elastic properties of the fiber. The first step of their study is to relate the distribution of stresses along linker DNA to the "macroscopic" stresses (force and torque) applied to the fiber ends. This allows to compute analytically the elastic constants describing the linear response of the fiber from the knowledge of naked DNA elastic constants, and to investigate quantitatively how they vary with the relaxed fiber structure, itself controlled by the "microscopic" structural parameters, as linker length $l$, or equivalently its relaxed twist $\tau^{0}$, and entry/exit angle $\Phi$ of linker DNA on the nucleosome [10]. The results substantiate an effective continuous description of the 30-nm fiber as an extensible worm-like rope (EWLR) extending the classical worm-like chain model [14] to an extensible and twistable chain 15 16 [17. In Section V, numerical results are presented and their physical meaning and implications are discussed. We then enlight our analytical results by comparing them with experimental results obtained by Cui and Bustamante [6] by pulling a single chromatin fiber. Section VI presents the biological relevance of our study. In particular, we exploit our complete and quantitative analysis to discuss how the elastic properties of the chromatin fiber might at the same time 
favour DNA compaction into the chromosomes and allow local decondensation of chromatin involved in gene expression.

\section{ASSEMBLY OF A MODEL CHROMATIN FIBER}

Chromatin is composed of a double-stranded DNA molecule wrapped from place to place around histone cores [3]. We here focus on the fixed-nucleosome case, corresponding to deacetylated histone tails [9]. This allows to model separately the nucleosomes and the linkers, i.e. the naked-DNA segments connecting the successive nucleosomes. Our model amounts to build step-by-step, i.e. nucleosome after nucleosome, a chromatin fiber. It incorporates aknowledged data on DNA structure, its bend and twist persistence lengths, and on the nucleosome structure. Each step of the assembly is described analytically, but we implemented this model within a Maple program in order to handle an arbitrary number of nucleosomes and to perform a quantitative analysis of the $\mathrm{SH}$ geometric properties.

\section{A. Linker modeling}

Linker DNA is in the usual B-DNA form: it is a righthanded double-helix, of radius $a \approx 1 \mathrm{~nm}$ and pitch equal to $l^{0}=3.4 \mathrm{~nm}$, which corresponds to $n_{b p}^{0}=10.6$ base pairs (bp) per turn. We shall suppose in the following that all the linkers have the same number $n_{b p}$ of base pairs, i.e. the same length $l=n_{b p} l^{0} / n_{b p}^{0}$. It corresponds in vivo to phased nucleosomes observed for instance in satellite chromatine [18. It corresponds in vitro to reconstituted fibers, involving repeated sequences each having a strong positioning effect on nucleosomes [19] [20]. In fact, we shall need this hypothesis to be satisfied only locally, over a few linkers, so that our model also accounts for irregular native chromatin.

Since we are looking for generic properties of the chromatin fiber, originating from its assembly, sequence effects are ignored (besides, they might be treated in a second stage, within the same modeling, as local strains superimposed to the relaxed homogeneous structure that we here consider). Without sequence effects, the linker is straight in absence of applied constraints; experimental evidence for straight linkers in relaxed fibers supports our generic modeling [21].

Linker DNA will be considered as an homogeneous cylindrical rope of radius $a$, that may twist and bend, but not stretch: due to the large value of the stretch modulus $\gamma_{D N A} \approx 1200 \mathrm{pN}$ [15], 22, stretch energy and strain can be ignored in linker DNA, at least in the low-stress situations that will be considered here (forces below $20 \mathrm{pN}$ ). Linker DNA is thus seen as a non extensible semi-flexible polymer and described within the continuous worm-like rope model (worm-like chain model supplemented with twist energy [12]); the elastic energy densities (energies per unit length) thus write:

$$
\begin{array}{rlrl}
\epsilon_{\text {twist }} & =\frac{k_{B} T C}{2}\left(\omega-\omega^{0}\right)^{2} & C & =75 \mathrm{~nm} \quad \text { (twist) } \\
\epsilon_{\text {bend }}=\frac{k_{B} T A}{2} \rho_{D N A}^{2} & A=53 \mathrm{~nm} \quad \text { (bend) }
\end{array}
$$

where $T$ is the temperature, $k_{B}$ the Boltzmann constant, $\rho_{D N A}$ the local curvature of the constrained linker and $\omega$ the twist rate. The relaxed twist rate $\omega^{0}=2 \pi / l^{0}$ is supposed to be homogeneous, which again amounts to ignore sequence effects. The constants $A$ and $C$ are respectively the bend and twist persistence lengths of DNA, here given for $10 \mathrm{mM} \mathrm{NaCl}$ (or any other monovalent salt) 23] [11] [12]. We expect $C$ to be almost independent of the ionic strength, since twist constraints are not strongly affected by electrostatics. By contrast, the electrostatic contribution to $A$ is important 24] 25]. The non-electrostatic contribution provides a lower bound $A \geq 23 \mathrm{~nm}$, which is in fact an overestimate; the experimental lower bound is rather $A \geq 40 \mathrm{~nm}$ [26].

We choose a direction along the DNA path corresponding to increasing indices $j$. We denote $s \in[0, l]$ the arclength along the dsDNA axis. Geometrically, the linker $j$ is described as represented on Figure 1 by the local axis $\vec{u}_{j}(s)$ of the double helix and a vector $\vec{t}_{j}(s)$, locally tangent to the minor groove and rotating around $\vec{u}_{j}(s)$ with an angular rate $\omega \cdot \vec{t}_{j}(s)$ accounts for the torsional state of the linker: the angle of the rotation transforming $\vec{t}(0)$ into $\vec{t}(l)$ is precisely the twist $\tau=l \omega$ of the linker. The vectors $\vec{u}_{j}(s)$ and $\vec{t}_{j}(s)$ are normalized to 1 and make a constant angle: $\vec{u}_{j}(s) \cdot \vec{t}_{j}(s)=\sin \left(\alpha_{D N A}\right)$ independent of $j$ and $s$. The slope of the strands in the double helix writes $\tan \left(\alpha_{D N A}\right)=l^{0} / 2 \pi a$, hence $\alpha_{D N A} \approx 28^{\circ}$. The vector $\vec{u}_{j}(s)$ is independent of $s$ in the relaxed fiber (a variation with $s$ would describe the linker bending in constrained state), whereas $\vec{t}_{j}(s)$ rotates around $\vec{u}_{j}$ with the relaxed twist rate $\omega^{0}$.

\section{B. Nucleosome modeling and assembly of the chromatin fiber}

Nucleosome structure is now well-known, thanks to high resolution cristallography data 113. Histone tails lock DNA on the histone core, so that nucleosomes are fixed on the DNA and the net effect of the nucleosome on the DNA trail can be described as a rigid kink connecting the linkers $j$ and $j+1$. In consequence, twisting the linker $j$ of a given angle reflects in a rotation of the same angle of the linker $j+1$ around the linker $j$. The rotational positioning of nucleosome $j+1$ with respect to nucleosome $j$ is thus entirely prescribed by the twist angle $\tau=l \omega$. In the relaxed state, it is equivalently prescribed by the linker length $l\left(\omega=\omega^{0}, \tau^{0}=l \omega^{0}\right)$. 
Technicalities about the assembly and its geometric description are presented on Figure 2. The linker $j$ enters the nucleosome $j$ at an entry point $E_{j}$ well-localized on the histone core. The linker DNA is grafted to the nucleosome by its minor groove [13]; this implies that the plane spanned by $\vec{u}_{j}$ and $\vec{t}_{j}(l)$ prescribes the position and the orientation of the nucleosome, given its radius $r_{n u c l}=5.5 \mathrm{~nm}$, its height $H=5.7 \mathrm{~nm}$ and the angle $\alpha=4.47^{\circ}$ between the linker and the nucleosome axis $\vec{N}_{j}\left(\vec{u}_{j} \cdot \vec{N}=\sin \alpha\right)$. As shown in Figure 2 (right), $\vec{u}_{j}$, $\vec{t}_{j}(l)$ and $\vec{N}_{j}$ belong to the same plane, which is nothing but the tangent plane to the nucleosome at $E_{j}$. Similarly, the plane tangent to the nucleosome $j$ at the exit point $S_{j}$ where linker $j+1$ leaves the nucleosome is spanned by $\vec{u}_{j+1}$ and $\vec{t}_{j+1}(0)$, which thoroughly determines this outgoing linker. The relative positioning of the linkers is conveniently described by the angle $\Phi$ ( $\Phi>0$ by convention) between their projections on a plane perpendicular to the nucleosome axis $\vec{N}_{j}$. The structural effect of nucleosome $j$ amounts to a translation in space from the entry point $E_{j}$ to the exit point $S_{j}$ and a rotation of an angle $4 \pi-\Phi$ around the nucleosome axis $\vec{N}_{j}$, transforming $\left(\vec{u}_{j}, \vec{t}_{j}(l)\right)$ into $\left(\vec{u}_{j+1}, \vec{t}_{j+1}(0)\right)$. As long as we consider an homogeneous fiber, $\Phi$ is independent of $j$. The line passing through the nucleosome center $G_{j}$ and directed along the bissector $\vec{D}_{j}$ of this angle $\Phi$ is the so-called dyad axis of the nucleosome $j$. Experimental evidence that the entry-exit angle $\Phi$ varies with salt concentration (it contracts when salt concentration increases) 19] 21] 28] 27 motivates us to study various values of $\Phi$. We here ignore interactions between nucleosomes. This is valid in low-salt conditions, in which the chromatin is expected to be extended, with internucleosomal distances larger than the interaction range. In high-salt conditions, our model aims at describing the "geometric" contribution to chromatin elastic behavior, i.e. the contribution originating from the DNA elastic properties and relayed by the chromatin fiber architecture. Comparison with observed elasticity would give access to the neglected interactions.

\section{Linker histones and chromatosome modeling}

In vivo and in vitro experiments show that the condensed conformations of the chromatin fiber are obtained for a more complex assembly of DNA and histones: an additional histone $\mathrm{H} 1$ (or a close chemical variant $\mathrm{H}$ 5, for instance in chicken erythrocytes) is bound to linker DNA near its entry/exit site on the nucleosome 21] [29]. Presumably, the role of this "linker histone" is both to secure the wrapping of DNA around the nucleosome and to stabilize the DNA helical coiling forming the $30-\mathrm{nm}$ fiber [30] 31]. Digestion experiments evidenced that the "core particle" now involves 166-168 bp of DNA (among which the 146 bp wrapped around the histone octamer); this new, larger, entity is called a chromatosome. The position of linker histone with respect to linker DNA and nucleosome, as well as the wrapping of DNA around it, are still debated 29] 31. It is only recognized that 166$168 \mathrm{bp}$ are protected and that the angle (still denoted $\Phi)$ between ingoing and outgoing linker DNA is lowered, varying with ionic strength. Our model easily accomodates the presence of linker histones along the fiber since only the resulting kink of DNA path is to be described. We may roughly account for the presence of linker histone by modifying $\Phi=130^{\circ}$ (without linker histone) into $\Phi=90^{\circ}$ at low salt down to $\Phi=45^{\circ}$ in high salt 27] 31]; the nucleosome is then replaced, within the same two-angle modeling, by an effective cylindrical chromatosome, with possibly different values for $r_{n u c l}, H$ and $\alpha$. For instance $r_{n u c l}=0$ and a lower value of $H$ would reproduce the crossing of ingoing and outgoing linkers in the neighborhood of the linker histone. Also, the effective length of the linkers is to be reduced by 20-22 bp.

We might thus study within the same geometrical modeling all the different instances encountered for the nucleosome: basic nucleosome or nucleosome dressed with $\mathrm{H} 1$, with or without chemical modification of $\mathrm{H} 1$ tails. The specific biological details ( $\mathrm{H} 1$ positioning, possible acetylation of histone tails [32]) would be taken into account first in $\Phi$, whose value is experimentally accessible, then in a second step by a precise fit of the parameters $r_{n u c l}$, $H$ and $\alpha$ of the effective cylindrical "core particle", so as to reproduce accurately the kink induced in the linker DNA broken line. Nevertheless, a systematic study led us to think that $\Phi$ is a far more important control parameter than $r_{n u c l}, H$ and $\alpha$, which will be henceforth considered as fixed characteristics.

To summarize, the parameters of the model, to be varied in our study, are the two angles $\tau^{0}$ (or equivalently $l=\tau^{0} / \omega^{0}$ or $\left.n_{b p}\right)$ and $\Phi$. The angle $\tau^{0}$ determines the relative positioning of two successive nucleosomes, whereas the angle $\Phi$ determines the relative positioning of two successive linkers.

\section{THE CHROMATIN SUPERHELIX (SH)}

The regularity of the microscopic assembly enforces an helical organization: we shall speak of the chromatin superhelix (SH). We now describe the main geometric properties of this helical coiling resulting from the regular assembly of linkers and nucleosomes. As shown in Figure 3 , our qualitative structural predictions recover standard structures proposed yet long ago 33] 34. In particular, we note that the various structures obtained when varying $\tau^{0}$ (or equivalently $n_{b p}$ ) and $\Phi$ cover both ribbon structures (less than 3 nucleosomes per turn) and crosslinked structures (more than 3 nucleosomes per turn). We avoid to speak of "solenoid" structure as this term refers to a model with bent linkers [35], whereas our modeling considers that linkers are straight in the relaxed 
fiber.

\section{A. Symmetry properties of the SH}

We first underline the symmetry properties of the SH, of much importance since they will reflect in the elastic properties of the fiber in the linear regime. The $\mathrm{SH}$ exhibits a discrete helical invariance: all the linkers (respectively all the nucleosomes) are equivalent, up to a rotation of angle $\theta$ around the axis $\vec{A}$ of the SH and a translation along this axis, bringing the linker $j$ onto the linker $j+1$ (respectively the nucleosome $j$ onto the nucleosome $j+1)$. The nucleosome centers all lay on a cylinder of axis $\vec{A}$ and radius $R$, whereas the entry and exit points all lay on a cylinder of radius $r$ (see Figure 4).

As the two strands of dsDNA are oriented in opposite directions (see Figure 1), there is no privileged orientation along linker DNA and only the nucleosome geometry can break the symmetry upon reversal of the fiber. Hence the fiber is not oriented provided the entry and exit points in a nucleosome have the same characteristics: it is the case in absence of linker histone, or when linker histone is positioned symetrically with respect to $E_{j}$ and $S_{j}$. The fiber properties are then unchanged under the transformation:

$$
\begin{aligned}
j & \longleftrightarrow N+1-j \\
S_{j} & \longleftrightarrow E_{N+1-j} \\
\vec{u}_{j} & \longleftrightarrow-\vec{u}_{N+1-j}
\end{aligned}
$$$$
\begin{aligned}
\vec{N}_{j} & \longleftrightarrow-\vec{N}_{N+1} \\
\vec{A} & \longleftrightarrow-\vec{A} \\
P_{j}(s) & \longleftrightarrow P_{N+1-j}
\end{aligned}
$$

Here $j=0 \ldots N+1$ where $N$ is the number of nucleosomes in the fiber beginning in $S_{0}$ with linker 1 and ending with linker $N+1$, at $E_{N+1}$ It follows that the nucleosome axes are all tangent to the cylinder containing the nucleosome centers, and that the line $\vec{D}_{j}$ relating the nucleosome center $G_{j}$ to its orthogonal projection onto the $\mathrm{SH}$ axis is nothing but the dyad axis of the nucleosome $j$ (see Figure 5, left). This dyad axis is invariant under the above reversal transformation. When $\tau^{0}$ is varied, the linker direction $\vec{u}_{j}$ sweeps a cone of axis $\vec{D}_{j}$ (or $\vec{D}_{j-1}$, due to the symmetry upon reversal of the $\mathrm{SH}$ ) and angle $\xi$, depending on $\Phi$ (see Figure 5, right). This property simply reflects the fact that the geometry of the fiber assembly is thoroughly determined once the nucleosome is positioned with respect to the $\mathrm{SH}$ axis, due to the symmetry properties and to the fact that the junction between the nucleosome and the ingoing and outgoing linkers is a rigid kink. Indeed, by symmetry, the nucleosome dyad axis $\vec{D}_{j}$ is orthogonal to the SH axis $\vec{A}$ and points towards it; the remaining "degree of freedom" is the orientation of the nucleosome axis $\vec{N}_{j}$ in the plane orthogonal to $\vec{D}_{j}$; when $l$ varies, $\vec{N}_{j}$ rotates together with $\vec{u}_{j}$ and $\vec{u}_{j}+1$ around $\vec{D}_{j}$. This orientation is thoroughly described by the angle $\beta \in\left[0,2 \pi\left[\right.\right.$ between its axis $\vec{N}_{j}$ and the SH axis $\vec{A}$, in the frame $(\vec{A}, \vec{D}, \vec{A} \wedge \vec{D})$. This angle satisfies $\cos \beta=\vec{N}_{j} \cdot \vec{A}$.

\section{B. SH geometric characteristics}

The relevant quantities to be computed (lengths $D$ and $r$, angles $\theta, \beta, z, \eta$, and $\xi$ ) are shown on Figures 4,5 and 6. All these quantities are independent of $j$, due to the (discrete) rotational invariance of the $\mathrm{SH}$. When linker lengths are all increased by the DNA pitch $l^{0}$, the twist angle of each linker increases by $2 \pi$, hence the relative orientations of successive linkers, or successive nucleosomes, are unchanged. It follows that the $\mathrm{SH}$ shape is preserved: the angles (as $z, \eta$ or $\theta$ ) are unchanged whereas the length $r$ is scaled by a factor $\left(1+l^{0} / l\right.$ ) (when comparing $l$ and $\left.l+l^{0}\right)$; the lengths $R_{S H}$ and $D$ are also scaled, but the involved scaling factor is smaller, since the contribution of the nucleosomes to the actual size of the $\mathrm{SH}$ is the same for $l$ and $l+l^{0}$; the precise value of this factor moreover depends on the structure of the SH (nucleosome orientation, for instance).

We here underline two points. First, the orientation of the $\mathrm{SH}$ axis is chosen so that the distance $D$ between two successive nucleosomes along the $\mathrm{SH}$ axis $\vec{A}$ is positive: $D>0$ (i.e. $D=\vec{A} \cdot\left[G_{j} \vec{G}_{j+1}\right]>0$ by definition of $\vec{A}$ ). Also, since the structure is discrete, the angle $\theta$ of the rótation $\mathcal{R}_{\theta}$ around the SH axis $\vec{A}$, transforming the projection of a nucleosome onto the plane orthogonal to $\vec{A}$ thto the following one is not univoquely defined: $\theta_{0}>0$ and $\theta_{0}-2 \pi<0$ are both possible (think to the array of grains in a corn ear or to the cells on a pineapple peel). By convention, we choose the value of smallest modulus, hence let $\theta$ vary in ] $-\pi, \pi]$; it corresponds to the (projected) angle swept by the geodesic (the array of grains of largest slope) relating $G_{j}$ to $G_{j+1}$ onto the cylinder of axis $\vec{A}$ and radius $R$.

$\Delta=\operatorname{dist}\left(G_{1}, G_{2}\right)$ and the angle $\Psi=\widehat{G_{1} G_{2} G_{3}}$ defined by three The pitch $P$ of the SH is given by $P=2 \pi D /|\theta|$. Note that the choice of the value of smallest modulus for $\theta$ is essential to get the actual value of the pitch through this formula. The degree of compaction can be measured as the number of nucleosomes per $10 \mathrm{~nm}$ of fiber, equal to $10 / D$ when $D$ is given in $\mathrm{nm}$.

\section{Excluded-volume effects}

Excluded volume (of nucleosomes but also linkers) has to be taken into account to discard unrealistic structures. In consequence, the above results should be supplemented by a geometric condition assessing whether they actually correspond to a possible structure, i.e. compatible with excluded-volume constraints. We here rather state an upper bound, involving only the geometric parameters of the fiber. Recall that $\beta$ is the angle 
between the nucleosome axes $\vec{N}_{j}$ and the SH axis (independent of $j$ by symmetry). A sufficient condition ensuring that no steric hindrance is encountered in the $\mathrm{SH}$ structure is that $P>P_{c}$ where:

$$
P_{c}=H \cos \beta+2 R|\sin \beta|
$$

where $R$ is the distance of the nucleosome centers to the $\mathrm{SH}$ axis (computed in the Maple implementation) and $H$ the height of a nucleosome. For $\beta=0$, this upper bound gives the exact threshold $P_{c}=H$. All linker lengths such that $P(l, \Phi) \leq P_{c}$ are forbidden. Note that a local breaking of this criterion is allowed: it is still possible that a few linkers lengths (less than $2 \pi / \theta$ ) take this value. A more accurate check can be obtained by drawing for each configuration the developped representation of the $\mathrm{SH}$, i.e. the mapping on a plane of the fiber track on the cylinder of radius $R$, as shown on Figure 7 . Its construction is straightforward, knowing the geometric characteristics $D, \theta$ and $\beta$. Excluded-volume constraints are satisfied if the tracks of any two nucleosomes do not intersect. This method requires to check each configuration, hence comes after the criterion on $P-P_{c}$, when it fails.

\section{Numerical results}

The major interest of our numerical implementation is to go further than a qualitative description and to compute explicitly any geometric characteristic of the relaxed fiber, in order to study quantitatively its variation with $l\left(\right.$ or $\tau^{0}$ ) and $\Phi$. The main results are presented in Figures 8,9 and 10 for a linker length between 30 and 50 bp (usual case). The quantitative geometric characterization of the $\mathrm{SH}$ is essential to compare the different structures obtained for different values of $(l, \Phi)$. It is also interesting from an experimental viewpoint, since it would allow to extract informations on the chromatin assembly from the experimentally accessible values of $D$ and $\theta$.

We first evidence that a regular helical packing of nucleosomes is compatible with straight linkers and does not require nucleosome-nucleosome interactions (but the special configurations exhibiting columnar arrays of nucleosomes are likely to be stabilized by such internucleosomal interactions). Mainly, our systematic analysis shows that it always lead to a 30-nm fiber. Although the detailed structure of the fiber is strongly sensitive to linker length $l$ and entry/exit angle $\Phi$, the value of about $30 \mathrm{~nm}$ for the fiber diameter $2 R_{S H}$ is a robust feature, hence of low structural significance; for instance, it does not discriminate close cross-linked structures and more extended ribbons;

We enlight some remarkable geometries (see also Section V.E):

- a ribbon - or zigzag - structure is obtained for $\theta=\pi$, when the number of nucleosomes per SH turn reaches the minimal value 2 ; then all the nucleosome dyad axes are aligned, $\beta$ is close to $\pi / 2$ but slightly different: $\beta=$ $\pi / 2-\alpha$ (see Figure 14).

- a particular packing is obtained when $n_{b p} / n_{b p}^{0}$ equals an integer; the nucleosome axes are then all aligned, hence all parallel to the SH axis: $\beta=0$. A fine tuning of $n_{b p}$ or $\Phi$ brings then into columns as seen on Figure 3 .

- the vanishing of the pitch $P$ is accompanied by a reversal of the SH axis, from which follows that $\cos z$ exhibits a jump from a value $c$ to the value $-c$ and $\theta$ jumps to the value $2 \pi-\theta$. We shall see that the vanishing of the pitch $P$ is an important (although virtual) event, having striking implications on the elastic properties of the fiber. - a change of chirality is observed for $\eta=\pi$ : the $\mathrm{SH}$ is right-handed for $\eta \in[0, \pi]$ and left-handed for $\eta \in[\pi, 2 \pi]$. For $\eta=\pi$, linkers cross the $\mathrm{SH}$ axis.

One has to carefully distinguish between:

- the handedness of the track passing through the nucleosome centers. As we have seen above in the definition of $\theta$, the handedness of this discrete structure is ill-defined. A same architecture can be seen as right-handed (direct rotation $\mathcal{R}_{\theta}$ ) or left-handed (indirect rotation $\mathcal{R}_{\theta-2 \pi}$ ). The choice of the value of smallest modulus, adopted here, corresponds to the shortest path. This "pseudochirality" is then given by the sign of $\theta$ and changes when $\theta$ crosses the value $\pm \pi$ or when $D$ (or equivalently the pitch $P$ ) vanishes. We underline that this structural feature does not define a relevant chirality, due to the arbitrariness of its definition, and should not be confused with the chirality of the SH, defined as follows;

- the chirality of the linker DNA trail (a broken line); it is determined by the position of $\eta$ with respect to $\pi$, i.e. by the sign of $\cos (\eta / 2)$. As fiber elasticity originates from linker elasticity, this chirality is the only relevant one for our mechanical study and will be henceforth adopted as the definition of the fiber chirality. We expect this chirality to determine the coupling between the twist and stretch elastic degrees of freedom i.e. the sign of $g$ to coincide with the chirality.

In the same spirit, we warn about the difference between the fiber and an helical DNA coiling passing through the nucleosome centers, in particular in what concerns their mechanical properties.

One of the conclusions of this thorough structural study is the fact that the connection between the microscopic parameters and the SH geometric characteristics is too complex and multivariate to get small-scale informations from structural observations of the fiber. We underline that the study of chromatin structure is not in itself sufficient to unravel its biological functioning, all the more as structural observation of the fiber is difficult and some results questionable [36]. Moreover, structural changes might not be the only way to pass local DNA modifications to higher scales; the functioning might rather be controlled by elastic properties.

For these two reasons, we turn to the analysis of the mechanical properties of the fiber, which are now at hand 
since we know explicitly all the geometric characteristics of the fiber assembly.

\section{ANALYTIC CALCULATION OF THE ELASTIC CONSTANTS OF THE CHROMATIN FIBER}

\section{A. Modeling the chromatin fiber as an extensible worm-like rope (EWLR)}

Our aim is to study the elastic response of the fiber to external stresses at scales larger than its pitch. We thus consider that we apply to the fiber a force $\vec{F}$, along the fiber axis $\vec{A}$; if $\vec{F}$ were not directed along the $\mathrm{SH}$ axis, it would induce a transitory motion, compelling the axis to align itself with the force direction, at least far from the ends. We here only suppose that this constrained equilibrium is yet reached. We may also apply a torsional torque $M_{t}$ (directed along $\vec{A}$ ) and a flexural torque $\vec{M}_{b}$, i.e. a torque component orthogonal to $\vec{A}$. Computation of elastic coefficients describing the linear response of the fiber to the applied force and torque will be performed analytically within a continuous description of the fiber, i.e. an effective large scale description in which the discrete nature of the assembly is smoothed out. We denote $S$ the arclength along the axis of the relaxed $\mathrm{SH}, u(S)$ its local relative extension, $\Omega(S)$ its local twist rate and $\varrho(S)$ its local curvature, with respect to the straight and untwisted relaxed state of the fiber for which $u, \Omega$ and $\rho$ thus identically vanish. Due to the axial symmetry of the relaxed $\mathrm{SH}$ at scales larger than its pitch, the bending energy density does not depend on the direction of the bending so that it involves only the total curvature $\rho$ and the modulus $M_{b}$ of the flexural torque (the special instance of ribbon-like configurations that break this axial symmetry is discussed below in Section $\mathrm{V}-\mathrm{E}$ ). The fiber has thus only three degrees of freedom $u, \Omega$ and $\rho$, local along the fiber. These strains $u, \Omega$ and $\rho$ are the canonical variables of the density of elastic free energy, which reflects in the following differential form:

$$
d \epsilon_{S H}=F d u+M_{t} d \Omega+M_{b} d \rho
$$

We restrict to the linear response regime, which expresses in the following relation:

$$
\left(\begin{array}{l}
F \\
M_{t} \\
M_{b}
\end{array}\right)=\left(\begin{array}{ccc}
\gamma & k_{B} T g & 0 \\
k_{B} T g & k_{B} T \mathcal{C} & 0 \\
0 & 0 & k_{B} T \mathcal{A}
\end{array}\right)\left(\begin{array}{l}
u \\
\Omega \\
\rho
\end{array}\right) \equiv \Gamma\left(\begin{array}{l}
u \\
\Omega \\
\rho
\end{array}\right)
$$

where it can be shown that the stress-strain tensor $\Gamma$ is necessarily symmetric (a special instance of Onsager relations). This linear response ansatz relates the strains $u, \Omega$ and $\rho$ of the fiber and the stresses $F, M_{t}$ and $M_{b}$ experienced by the fiber. $\mathcal{A}$ is the bend persistence length of the fiber, $\mathcal{C}$ its twist persistence length, $\gamma$ its stretch modulus (dimension of a force) and $g$ the twist-stretch coupling constant (no dimension).

Plugging the linear response ansatz (5) into (4) leads to the SH density of elastic free energy:

$\epsilon_{S H}(S)=\frac{k_{B} T \mathcal{A} \varrho^{2}(S)}{2}+\frac{k_{B} T \mathcal{C} \Omega^{2}}{2}+\frac{\gamma u^{2}(S)}{2}+k_{B} T g \Omega(S) u(S)$

Such a continuous description of the fiber can be termed "extensible worm-like rope" model (EWLR) [16]. It extends the WLC model introduced in 1949 by Kratky and Porod and currently used to describe stiff polymers $\sqrt{14}$ by accounting for twist (as above for linker DNA [12]) but also stretch degrees of freedom. The fact that chromatin is chiral demands a linear coupling between twisting and stretching. Due to the axial symmetry of the $\mathrm{SH}$, $\left(\vec{F}, \vec{M}_{t}, \vec{M}_{b}\right)$ and $\left(\vec{F}, \vec{M}_{t},-\vec{M}_{b}\right)$ (and also $\left(\vec{F}, \vec{M}_{t}, \mathcal{R} \vec{M}_{b}\right)$ where $\mathcal{R}$ is any rotation around the axis $\vec{A}$ leaving the $\mathrm{SH}$ unchanged) should induce the same energy change in the SH. This symmetry argument shows that there is no other coupling term at the linear order considered here [15]. This model has been fully investigated by many groups in the context of DNA; in particular, forceextension curves have been obtained [16]; a nonlinear term $V(u)$ might be added in (6) to go beyond the linear response regime. These results can be straightforwardly transposed to chromatin fiber, so that the description of the harmonic elastic behavior of the chromatin fiber reduces to the computation of the four elastic coefficients $\mathcal{A}, \mathcal{C}, \gamma$ and $g$ involved in the EWLR model. Their determination from the computation of the elastic energy stored in the constrained linkers will prove that the fiber actually fits in an EWLR model when considered at large enough scale.

\section{B. A general analytic method for computing elastic coefficients}

The first aim of our study is to express the elastic coefficients $\mathcal{A}, \mathcal{C}, \gamma$ and $g$ as a function of the elastic coefficients of linker DNA, given the relaxed geometry of the fiber. A key point of our approach is to relate the stresses exerted respectively on the linker, considered as a WLR, and on the fiber, considered as an EWLR instead of relating the strains $\left(\omega-\omega^{0}, \rho_{D N A}\right)$ of the linkers and the strains $(u, \Omega, \rho)$ arising at the fiber level (this approach appears to be technically cumbersome and analytically intractable, see Section VII.B). Although the stresses $F$, $M_{t}$ and $M_{b}$ are not the canonical variables of the free energy density $\epsilon_{S H}$, we shall express it as a function of these variables $F, M_{t}$ and $M_{b}$. Plugging (5) into (6) leads to the following expression of the free energy density:

$$
\epsilon_{S H}=\frac{\left[k_{B} T \mathcal{C} F^{2}+\gamma M_{t}^{2}-2 k_{B} T g F M_{t}\right]}{2\left(k_{B} T \mathcal{C} \gamma-k_{B}^{2} T^{2} g^{2}\right)}+\frac{M_{b}^{2}}{2 k_{B} T \mathcal{A}}
$$


The principle of the computation is the following: knowing the relaxed geometry of the fiber, i.e. the quantities $r, \eta, z$ and $D$ computed in Section III, it is possible to analytically determine the local stresses experienced by linker DNA when the fiber is constrained at its ends (and only at its ends) and to deduce the elastic energy of a linker as a quadratic function of $\left(F, M_{t}, M_{b}\right)$. The identity of the elastic energy of the fiber and the elastic energy stored in the linkers will allow a termwise identification with (7), which leads the values of $\mathcal{A}, \mathcal{C}, \gamma$ and $g$ as analytical functions of the geometric parameters of the fiber, and through our numerical implementation, as a function of $l$ (or $\tau^{0}$ ) and $\Phi$.

A much important intermediate result is to establish the relation between the local stresses (experienced by the linkers) and the global stresses (applied on the chromatin fiber). The current point on linker $j$, with arclength $s$, is denoted $P_{j}(s)$. Recall that linker $j$ leaves the nucleosome $j-1$ at $S_{j-1}$ and enters the nucleosome $j$ at $E_{j}$ (see Figure 6 ). We denote $\vec{f}_{j}(s)$ the force and $\vec{m}_{j}(s)$ the torque exerted at the point $P_{j}(s)$ of linker $j$ by the upstream part of the fiber. The main arguments involved in the derivation are the following:

- when only pulling the fiber, the rotational invariance of the relaxed fiber should be preserved when end effects are ignored. We may (and in fact should) focus on the universal, rotationally symmetric behaviour of the fiber, observed far from its ends. Indeed, the quadratic, rotationally invariant EWLR energy is to be fitted only to the quadratic, rotationally invariant energetic contribution coming from the linkers, otherwise the identification would not make sense.

- we restrict to the linear regime. We may consider separatedly the different stresses applied to the fiber and simply sum up their effects to recover the general solution. The contribution of the applied torque $\vec{M}$ to the local torque $\vec{m}_{j}(s)$ is merely $\vec{M}$.

The conclusion follows using standard equilibrium equations of spring mechanics. When external forces and torques are applied at the ends only, linear response hypothesis implies that the solution finally writes:

$$
\begin{aligned}
\vec{f}_{j}(s) & =\vec{F} \\
\vec{m}_{j}(s) & =\vec{M}-\left[O_{j}(s) \vec{P}_{j}(s)\right] \wedge \vec{F}
\end{aligned}
$$

where $O_{j}(s)$ denotes the orthogonal projection of $P_{j}(s)$ onto the $\mathrm{SH}$ axis $\vec{A}$. It gives the relation between the global stresses $\vec{F}$ and $\vec{M}$ exerted at the fiber ends and the local stresses experienced at the linker level, at each point of the DNA path. The rotational symmetry of the fiber ensures that $\vec{F}$ is directed along the SH axis $\vec{A}$ at equilibrium.

The term $\left[O_{j}(s) \vec{P} P_{j}(s)\right] \wedge \vec{F}$ reflects the involvement of

the fiber architecture in the expression of the local torque. As we restrict to the description of harmonic elasticity, the coefficients of $\vec{F}$ and $\vec{M}$ in the elastic energies of the linker will be computed within the relaxed $\mathrm{SH}$. We underline that we do not need to compute the constrained shape of the linkers to describe the linear response of the fiber to applied force and torque. We carry on the computation by exploiting the fact that the fiber is not oriented. It follows that: $\left\|O\left(\overrightarrow{E_{j}}\right) E_{j}\right\|=\left\|O\left(\overrightarrow{S_{j}}\right) S_{j}\right\|=r$ and:

$\left\|\left[O_{j}(s) \vec{P}_{j}(s)\right] \wedge \vec{A}\right\|^{2}=\left\|O_{j}(s) \vec{P}_{j}(s)\right\|^{2}=r^{2}\left[\cos ^{2}(\eta / 2) \cos ^{2} z+\sin ^{2}(\eta\right.$

where $\eta, r$ and $z$ are the values associated to the relaxed SH.

\section{Small-scale grounds for the EWLR modeling of the chromatin fiber}

Linear response ansatz applies both at the linker level (WLR model, with parameters $A$ and $C$ ) and at the $\mathrm{SH}$ level (EWLR model with parameters $\mathcal{A}, \mathcal{C}, \gamma$ and $g$ ). Knowing the stresses thus gives the strains, respectively $\left(\rho_{D N A}, \omega-\omega^{0}\right)$ and $(u, \Omega, \rho)$, if required, and the elastic energies. The computation of the $\mathrm{SH}$ elastic constants rests on the obvious but essential fact that the elastic energy stored in the SH, expressed by (7) at the SH level, is nothing but the sum of the elastic energies stored in its linkers, since no interactions are involved in our modeling.

A key point is the different decompositions of the torques into torsional and flexural components at the fiber level and at the DNA level:

$\vec{M}(S)=M_{t} \vec{A}+\vec{M}_{b}(S)$

$$
\vec{m}(s)=m_{t} \vec{u}+\vec{m}_{b}(s)
$$

The relation between the two decompositions involves the fiber geometry. We underline once more that in the linear response regime, only the relaxed geometry is involved. The first decomposition gives the components involved in the EWLR energy density whereas the second is required to compute the elastic energy of a linker. Indeed, the elastic energy densities of a linker can be expressed as a function of local stresses, according to:

$$
\left.\begin{array}{l}
m_{t}=k_{B} T C\left(\omega-\omega^{0}\right) \\
m_{b}=k_{B} T A \rho_{D N A}
\end{array}\right\} \Longrightarrow \epsilon_{\text {twist }}=\frac{m_{t}^{2}}{2 k_{B} T C} \quad \text { and } \quad \epsilon_{\text {bend }}=\frac{m}{2 k_{B}}
$$

According to a general result [5], besides easy to check directly here, $m_{t}$ is constant along the linker (i.e. independent of $s$ ) whereas $\vec{m}_{b}$ may vary with $s$ both in direction and modulus. 
The EWLR model is a continuous model. It makes sense to describe the elastic behavior of the discrete chromatin structure by means of an EWLR model only at large enough scale, so that the discrete effects are smoothed out. At lower scale, the specific orientation of each linker influences its energy, more precisely the contribution coming from the global stress $\vec{M}_{b}$, which breaks the rotational invariance; the persistence length of bending $\mathcal{A}$ of the $\mathrm{SH}$ is only defined after averaging over, say, one turn of SH. At the linker scale, twist-bend and stretch-bend coupling terms are present; they vanish on the average. The averaging keeps only the large scale, rotationally invariant, "EWLR-like" contribution. The remaining terms describe local contributions to the bending energy of the SH, cancelling each other, hence with no resulting effect at the fiber scale. The average over a number of linkers sufficient to recover the rotational invariance of the fiber (using $\frac{1}{N} \sum_{j=1}^{N} \vec{u}_{j} \sim(\cos z) \vec{A}$ ) is conveniently replaced (except for the ribbon-like structure for which the rotational invariance breaks down, see Section V-E) in the computation by an average over the directions of $\vec{M}_{b}$ (denoted $<>$ ): it has the same effect of extracting only the resulting contribution at large scale. After integration of the densities $\epsilon_{\text {bend }}$ and $\epsilon_{\text {twist }}$ along the linker, the elastic energies $E_{\text {bend }}$ and $E_{\text {twist }}$ stored in a linker write:

$$
\begin{aligned}
E_{\text {bend }} & =\frac{1}{2 A k_{B} T} \int_{0}^{l}<m_{b}^{2}(s)>d s \\
& =\frac{l F^{2} r^{2}}{6 A k_{B} T}\left(\sin ^{2}\left(\frac{\eta}{2}\right)+3 \cos ^{2} z \cos ^{2}\left(\frac{\eta}{2}\right)\right)+\frac{l M_{t}^{2}}{2 A k_{B} T} \\
E_{\text {twist }} & =\frac{1}{2 C k_{B} T} \int_{0}^{l}<m_{t}^{2}>d s=\frac{l<m_{t}^{2}>}{2 C k_{B} T} \\
& =\frac{l}{2 C k_{B} T}\left(F r \cos \left(\frac{\eta}{2}\right) \sin z+M_{t} \cos z\right)^{2}+\frac{l M_{b}^{2} \sin ^{2}}{4 C k_{B} T}
\end{aligned}
$$

\section{Analytic expression of the elastic constants as a function of geometric parameters of the fiber (SH)}

The quadratic expression of $E_{b e n d}+E_{\text {twist }}$ as a function of $F, M_{t}$ and $M_{b}$ is straightforwardly identified with (7) integrated over the length $D$. This justifies a posteriori to map the fiber and its elastic behaviour onto the continuous EWLR model. In other words, it provides a microscopic validation of the EWLR modeling of the chromatin fiber. In particular, we check the expected vanishing of the twist-bend and stretch-bend coupling. Some algebra finally yields the elastic constants of the SH fiber:

$$
\begin{aligned}
& \mathcal{A}=\frac{A D / l}{1-\frac{(C-A)}{2 C} \sin ^{2} z} \\
& \mathcal{C}=\frac{C D}{l}\left(\frac{\tan ^{2}(\eta / 2)}{3}+\cos ^{2} z+\frac{A}{C} \sin ^{2} z\right)\left(\frac{1}{1+\frac{\tan ^{2}(\eta / 2)}{3}\left(\frac{\cos ^{2} z+1}{32}\right.}\right. \\
& \gamma=k_{B} T \frac{D}{l}\left(\frac{A \cos ^{2} z+C \sin ^{2} z}{r^{2} \cos ^{2}(\eta / 2)}\right)\left(\frac{1}{1+\frac{\tan ^{2}(\eta / 2)}{3}\left(\frac{\cos ^{2} z+\frac{C}{A} \sin ^{2} z}{3 A}\right)}\right) \\
& g=\frac{D}{l}\left(\frac{(C-A) \sin z \cos z}{r \cos (\eta / 2)}\right)\left(\frac{1}{1+\frac{\tan ^{2}(\eta / 2)}{3}\left(\frac{\cos ^{2} z+\frac{C}{A} \sin ^{2} z}{3 A}\right)}\right)
\end{aligned}
$$

We underline that our approach yields the elastic constants of any relaxed geometry, hence allows an analysis of sensitivity with respect to $l$ (i.e. $\tau^{0}$ ) and $\Phi$.

\section{RESULTS AND PHYSICAL DISCUSSION}

\section{A. Numerical results for the elastic constants}

Numerical implementation of the above analytical formulas clandwerperformed for any valuekldete the microscopic

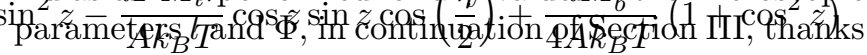
to a Maple program. We here present the results obtained in the two situations $\Phi=90^{\circ}$ and $\Phi=50^{\circ}$, taking (14) as a variable the linker length.

Starting from the microscopic structural parameters $(15)$ $\tau^{0}$ and $\Phi$, passing through the determination of the $\mathrm{SH}$ geometric characteristics $D, r, z$ and $\eta$, we obtain the explicit values of the elastic constants, as functions of $\tau^{0}$ (or equivalently $n_{b p}$ ) at fixed (arbitrary) $\Phi$. Indeed, expressions (16) are valid for any relaxed structure hence allow to analyze the complete range of variations of the elastic constants as $\tau^{0}$ and $\Phi$ vary, as shown in Figure 11. We here present two typical cases, currently proposed in chromatin structural studies: $\Phi=90^{\circ}$ then $\Phi=50^{\circ}$. A decrease of $\Phi$ is presumably induced by an increase of salt concentration, lowering the mutual repulsion of ingoing and outgoing linkers and strengthening the structural effect of linker histones. Experimental observations of a fiber dressed with linker histones showed that a value of $\Phi$ around $90^{\circ}$ corresponds to a salt concentration of less than $5 \mathrm{mM} \mathrm{NaCl}$, whereas $\Phi$ decreases below $50^{\circ}$ above $15 \mathrm{mM} \mathrm{NaCl}$ [19] [31].

The curves of Figure 11 evidence a strong sensitivity of elastic coefficients with respect to the fiber structure, as controlled by $n_{b p}$. A striking result of our study is the sharp decrease of all elastic coefficients together with the 
pitch $P$, around a critical value $n_{b p, c}(\Phi)$ for which $P$ vanishes. The critical value $n_{b p, c}(\Phi)$ depends on the value of $\Phi$ and more generally on the precise modeling of the chomatosome. As $P$ vanishes, steric hindrance between nucleosomes precludes to build a regular structure with $n_{b p}=n_{b p, c}(\Phi)$, so that this feature might be seen as irrelevant. Nevertheless, it is possible to have $n_{b p}=n_{b p, c}(\Phi)$ locally, i.e. over less than one turn of $\mathrm{SH}$; this flexibility, although local, might yet have dramatic consequences: it is possible to create a noticeable kink in the chromatine fiber at this point by applying only very weak stresses. Such "critical" turns appear as defects where the regular compact fiber is easy to "open". Moreover, the curves near $n_{b p, c}$ but above the excluded-volume threshold $P_{c}$ are still influenced by their vanishing in $n_{b p, c}$. The steep variation of the elastic constants in the neighborhood of $n_{b p, c}$ is an actual property, corresponding to a highly sensitive fiber: the geometric parameter $D$ and the elastic constants increase by a noticeable factor within one or two base pairs. Another important result, whose implications are discussed below (Section V-D), is the observed change of chirality of the SH, defined in Section III.D as the sign of $\cos (\eta / 2)$ and exactly correlated with the changes in the sign of the twist-stretch coupling $g$.

Finally, the relative energetic contribution of linker bending and linker twisting are shown on Figure 12 in pure cases where only one of the stresses $F, M_{t}$ or $M_{b}$ is applied (stretch, twist or bend at the fiber level). We checked that the stretching energy and the twist-stretch coupling energy of the linker are negligible (atmost a few percent of the total elastic energy stored in a linker) due to the high value of $\gamma_{D N A}$ (around $1200 \mathrm{pN}$ ) and $g_{D N A}$ (between 20 and 30) [15] 22]. The figure evidences an interesting feature coming from the spatial organization of DNA into a chromatin fiber. According to the fiber structure (i.e. linker length $l$ and angle $\Phi$ ) and to the nature of external stresses (pulling force, torsional or flexural torque) the linkers react either by bending, either by twisting, which might play a biological role by regulating the local DNA structure.

Our computation of $\mathcal{A}$ is based on its "energetic" definition through the expression (6) of the free energy of the EWLR description of the fiber: the energetic cost required for bending the $\mathrm{SH}$ axis and creating a uniform curvature $\rho$ is $k_{B} T \mathcal{A} \rho^{2} / 2$ per unit length. It originates from the cost of twist and bend distortions induced at lower scale on the linkers. This definition does not (and does not have to) consider possible steric hindrance (hard-core interactions). Indeed excluded-volume constraints do not contribute to elastic energies hence do not modify $\mathcal{A}$; they only forbid some deformations. To determine whether a given bending of the $\mathrm{SH}$ is possible, one has

- first to check whether such a bending is "geometrically" allowed, i.e. satisfied excluded-volume constraints ( a necessary condition is that $\rho R_{e x}<1$, i.e. the radius of curvature $1 / \rho$ should be greater that the excludedvolume radius $R_{S H}$ [37,

- secondly to check whether enough energy is available, using the value $\mathcal{A}$ determined above, notwithstanding the excluded-volume effects.

Note that these criterions are independent (geometric and energetic respectively) and they can be checked in any order.

\section{B. Comparison with an helical coiling (ordinary spring)}

It is interesting to compare the $\mathrm{SH}$ elastic behavior to that of a simple helical "spring" (a toroidal coiling of DNA), i.e. the continuous structure characterized by the same angle $z$ (here between its axis and the tangent to the elementary fiber) and the same radius $r$. The helical spring is thus implicitely controlled by the same parameters $l$ and $\Phi$ as the SH. The comparison enlights:

- that the chromatin fiber is actually a spring from a mechanical point of view, although a special, tunable, one;

- that the difference between ordinary springs and chromatin lies in the angle $\eta(\eta=0$ in ordinary springs whereas it is not small in chromatin), from which originates the tunable character of the chromatin spring.

The reasoning to compute the elastic constants of an helical spring is at each step analog to the above one (Section IV). We obtain the following expression for the average energies per unit length:

$$
\begin{aligned}
\epsilon_{t} & =\frac{\left(F r \sin z+M_{t} \cos z\right)^{2}}{2 C k_{B} T}+\frac{M_{b}^{2} \sin ^{2} z}{4 C k_{B} T} \\
\epsilon_{b} & =\frac{\cos ^{2} z F^{2} r^{2}}{2 A k_{B} T}+\frac{M_{t}^{2} \sin ^{2} z}{2 A k_{B} T}-\frac{F M_{t} r \cos z \sin z}{A k_{B} T}+\frac{M_{b}^{2}\left(1+\cos ^{2} z\right)}{4 A k_{B} T}
\end{aligned}
$$

where the average is performed either on the orientations of $\vec{M}_{b}$, either on a turn, i.e. over an arclength $2 \pi r / \sin z$ corresponding to the pitch $2 \pi r \cot z$. Such an average is essential to fit in the definition of elastic constants (continuous, rotationally invariant EWLR model). The expression of the elastic constants follows:

Ordinary spring

$$
\begin{aligned}
& \mathcal{A}=\frac{A \cos z}{1-\frac{(C-A)}{2 C} \sin ^{2} z} \\
& \mathcal{C}=\cos z\left(C \cos ^{2} z+A \sin ^{2} z\right) \\
& \gamma=\frac{k_{B} T \cos z}{r^{2}}\left(A \cos ^{2} z+C \sin ^{2} z\right) \\
& g=\frac{(C-A) \sin z \cos ^{2} z}{r}
\end{aligned}
$$


In fact, these classical formulas of spring mechanics can be recovered directly from those obtained for the chromatin $\mathrm{SH}$ by letting $l \rightarrow 0$ at fixed $z$ in the $\mathrm{SH}$ formulas; accordingly, $\eta \rightarrow 0$ and $D / l \rightarrow \cos z$. This link proves that chromatin is actually a special kind of spring. In the chromatin fiber, $\sin (\eta / 2)$ is not small and can even reach 1 . Hence all the features depending on $\eta$ will exhibit a striking difference when comparing the $\mathrm{SH}$ and the helical coiling of DNA. This is the angle $\eta \neq 0$, reflecting the discrete character of the chromatin assembly, which is mainly responsible of the tunable elasticity and tunable chirality of the chromatin fiber, as can be seen by comparing the elastic constants of the helical spring and of the chromatin fiber.

Let us also compare the distribution of DNA elastic energy between twist and bend degrees of freedom. The comparison is presented in Figure 12. When only a torque (either torsional, either flexural) is applied, the partitions in the $\mathrm{SH}$ case and in the case of an helical coiling are exactly identical. When only a pulling force is applied, the energy is mainly stored in the torsional energy in the case of a simple helical spring whereas it is the converse in the chromatin SH. In the chromatin fiber, the energy stored in the linker bending is in any cases larger (and sometimes far larger) than the energy stored in the twist. This remark reflects the following property of the SH: given $m_{t}$ and $m_{b}$ (modulus averaged along a linker), it is possible to determine a force $F$ and a torsional torque $M_{t}$ creating $m_{t}$ and $m_{b}$ on each linker only if $m_{b} \geq$ Cte. $m_{t}$, where the constant depends on the $\mathrm{SH}$ geometric characteristics. In particular, it is impossible to have $m_{b}=0$ unless $m_{t}=0$, i.e. $F=M_{t}=0$.

This difference between the elastic behavior of the $\mathrm{SH}$ and the corresponding simple helical coiling originates from the discrete structure of the $\mathrm{SH}$, where the linkers are spaced by rigid kinks (the nucleosomes). This strongly modifies the relation between the pulling force applied at the fiber ends and the local flexural $\left(m_{b}\right)$ and torsional $\left(m_{t}\right)$ components determining the DNA elastic energy. The discrepancy is maximal for $\sin (\eta / 2)=1$, which corresponds to the $\mathrm{SH}$ conformation in which the linkers cross the $\mathrm{SH}$ axis and $\mathrm{SH}$ chirality changes, which obviously never occurs in an helical coiling of bounded radius(either left-handed, either right-handed, but never in between if $r$ neither vanishes nor diverges). Although the SH " looks like" an helical coiling (see Figure 3), its response to a pulling force differs from that of an helical spring. Indeed, we again underline that its elastic properties are determined by the characteristics of the broken line formed by the linkers, which might be far different from an helical coiling (especially when $\eta$ is close to $\pi$, which corresponds to cross-linked structures).

Elasticity theory of an homogeneous cylindrical rod [5] 41] predicts the relation $4 \mathcal{A} k_{B} T=\gamma R_{S H}^{2}$. The predicted value of $\mathcal{A}$ using this formula is roughly correct for the helical coiling, but it overestimates the actual value of the $\mathrm{SH}$ persistence length by a factor of 2 or more. This discrepancy enlights the importance of the complex sub- structure of the SH, leading to a fiber far different from an homogeneously filled cylinder of radius $R_{S H}$ and even from an helical coiling of DNA.

\section{The origin of the sensitivity of the elastic constants with respect to the fiber structure}

Although the chromatin SH and an helical coiling of DNA exhibit quantitative differences, their elastic behaviors share the same key feature (see Figures 11 and 13): a sharp decrease towards 0 of the elastic constants, together with the pitch. Considering a simple helical spring yields the - quite intuitive - explanation of this feature: when the slope of the elementary (DNA) fiber decreases to 0 (DNA path almost orthogonal to the fiber axis), the number of turns per unit length along the fiber axis increases (see in particular the divergence of the compaction ratio $10 / D(\mathrm{~nm})$ on Figure 9, outside the figure frame). This accumulation of turns, each acting as an hinge, easily allows a huge deformation of the fiber, simply by the addition of small changes occuring in each turn. This explanation, involving only an accordion-like behavior, ensures the robustness of the feature. The flexibility dramatically increases when the number of coils per unit length of fiber increases, whatever the precise geometry with which the elementary fiber is coiled into a large-scale one.

The strong correlation between the pitch and the four elastic constants behaviors when $n_{b p}$ varies simply reflects in the very expression of the elastic constants: they all write as $(D / l) f(z, r)$, hence are slaved to the vanishing of $D$ (or $P$ ). The factor $D / l$ shows that the extensibility and flexibility of the $\mathrm{SH}$ are controlled by its degree of compaction. The more compact the conformation (low value of $P$ ), the more flexible is the fiber, easily bent, twisted or stretched. We claim that the result is typical, i.e. does not depend on the details of the modeling, but only on the general feature of the chromatin fiber assembly, i.e. nucleosomes linked by segments of DNA. This form $(D / l) f(z, r)$ also indicates that the elastic properties of the fiber are mainly controlled by the angle $z$ between the linkers and the SH axis. Indeed, z roughly determines the decomposition of local stresses among twist and bend degrees of freedom of linker DNA (stretch is always negligible).

\section{A multi-strand spring with tunable chirality}

We first underline that the chromatin fiber is a spring of tunable chirality: the sign of the twist-stretch coupling $g$, associated to the chiral nature of the $\mathrm{SH}$, changes twice within a "period" (an $l$-interval of width $10.6 \mathrm{bp}$, i.e. the DNA pitch), see Figure 11; correspondingly, a change from a right-handed $\mathrm{SH}$ to a left-handed SH occurs, as seen on Figure 1. Reverse behaviors are observed 
in right-handed and left-handed SH. Indeed, change of chirality exactly corresponds to change in the sign of the susceptibility $\partial D / \partial \tau$, i.e. the slope of the curve giving $D$ as a function of $n_{b p}$ (see Figure 8), up to a factor $\partial n_{b p} / \partial \tau=n_{b p}^{0} / 2 \pi$. The noticeable consequence is that a change $\Delta \tau$ will either decondense or compact the fiber according to the sign of $\partial D / \partial \tau$, i.e. to the chirality of the SH.

Far more, the two-level structure of the chromatin fiber makes it quite similar to a multi-strand spring: an interesting behavior arises from the interplay between the right-handedness of the linker DNA and the tunable chirality of its coiling within the $\mathrm{SH}$. Applying (only) a pulling force $F \vec{A}$ creates a torsional torque:

$$
m_{t}=\operatorname{Fr} \sin z \cos (\eta / 2)
$$

The sign of $\cos (\eta / 2)$ thus determines the sign of $m_{t}$ (recall that by definition $\sin z>0$ ). According to this sign, i.e. to the SH chirality, pulling the chromatin fiber will either twist, either unwind the linker DNA double helix. This behavior is well-known in the context of multistrand springs 38]. Its biological interest might be to regulate DNA denaturation required for transcription and replication. We get a similar result when applying a torsional torque $M_{t} \vec{A}$; the torsional torque at the linker level writes $m_{t}=M_{t} \cos z$. A change of $\operatorname{sign}$ in $\cos z$ inverts the action of the given torque $M_{t} \vec{A}$ at the DNA level, and we see that such a sign alternation actually occurs in each interval of length $10.6 \mathrm{bp}$.

Conversely, a modification of the linker twist, as can be achieved by intercalator enzymes, will modify in a tunable way the geometry of the SH fiber; according to the SH chirality, the same change of the linker twist will either condense $(\Delta D<0)$, either decondense $(\Delta D>0)$ the chromatin fiber. This possible mechanism of condensation/decondensation and its biological relevance will be investigated in a following paper (see also Section V.F).

\section{E. Special geometries}

Ribbon (or zigzag): a first peculiar geometry of the fiber is the zigzag or ribbon-like structure, for which the crosslinked and roughly toroidal coiling degenerates into a flat structure. The number of nucleosomes per turn then reaches its minimal value of 2 , i.e. $\theta=\pi$. In this case, represented on Figure 14,

$$
\beta=\frac{\pi}{2}-\alpha \quad \cos z=\sin \beta \sin (\Phi / 2)
$$$$
D=\frac{l \sin (\Phi / 2)}{\sin \beta}
$$

We locate this kind of configurations by determining on Figure 9 the linker lengths for which $2 \pi / \theta$ equals 2 . It appears (see Figure 10) that $\cos z$ then reaches is maximal value. Note that a ribbon is chiral, and is not the maximally extended configuration; on the contrary, a small length change $\left(\Delta n_{b p}=1\right)$ drives it into sterically forbidden configurations.

Nonchiral configurations: the SH chirality changes when $\overline{\cos (\eta / 2) \text { vanishes, i.e. } \mid} \eta \mid=\pi$; in this case, the linkers cross the $\mathrm{SH}$ axis. This configuration is remarkable mainly for its mechanical properties. Indeed, the absence of chirality amounts, from its very definition, to the vanishing of the twist-stretch coupling: $g=0$. Moreover, as explained in Section V.D, the sign of $\cos (\eta / 2)$ determines whether the linker DNA is unwound (i.e. denatured) or on the contrary twisted when the $\mathrm{SH}$ is pulled.

Since $\sin (\eta / 2)=1$, the configuration is the limiting case opposite to the helical spring, for which $\sin (\eta / 2)=$ 0 . In particular, when the fiber is only pulled $(\vec{M}=0)$ the twist energy stored in the linker vanishes: all the elastic energy of the linker is stored in the bending degree of freedom:

$$
E_{t}=0 \quad E_{b}=\frac{l F^{2} r^{2}}{6 A k_{B} T}=\frac{l^{3} F^{2} \cos ^{2}(\Phi / 2)}{24 A k_{B} T}>0
$$

which never occurs in an helical spring. We underline that non-chiral structures (special linker positioning) and ribbon-like structure (special nucleosome positioning) should not be confused.

In fact, this configuration is not rotationally symmetric, hence has two bend elastic constants. They are computed within the same lines as above, but without performing any average over the direction of the bending torque $\vec{M}_{b}$; the identification is done with an asymmetric EWLR $(4 \times 4$ matrix with two bend persistence lengths). This yields:

$$
\mathcal{A}_{1}=\frac{D A}{l} \quad \text { and } \mathcal{A}_{2}=\frac{D A}{l} \frac{1}{1-\frac{(C-A)}{C} \sin ^{2} z}
$$

Nevertheless, the orientation of the bending torque is not controlled at this level, hence the relevant elastic behavior, to be observed experimentally, is rather that predicted after having performed an average over the torque directions. This recovers a rotationally symmetric behavior, characterized by the above given persistence length $\mathcal{A}$, as it can be seen directly in the formula:

$$
\frac{1}{\mathcal{A}}=\frac{1}{2}\left(\frac{1}{\mathcal{A}_{1}}+\frac{1}{\mathcal{A}_{2}}\right)
$$

Columnar packing: special configurations are obtained when $n_{b p}$ is close to an integral multiple of $n_{b p}^{0}$ (for instance $n_{b p}=42 \mathrm{bp}$ or $43 \mathrm{bp}$ ). In this case, nucleosome axes are all parallel hence parallel to the $\mathrm{SH}$ axis due to the rotational symmetry. We actually check on the curves of Figure 10 that $\beta=0$ for $n_{b p}=42 \mathrm{bp}$, whatever 
$\Phi$. It is noticeable that geometric characteristics as $D, z$ and $\eta$ are much sensitive to variations of $n_{b p}$ around this value of $42 \mathrm{bp}$, which reflects in a similar sensitivity of the elastic constants, as seen on Figure 11. Fine tuning of $n_{b p}$ and $\Phi$ leads to a configuration in which the nucleosomes are organized in columnar arrays. It has been evidenced [39] 40] that free nucleosomes exhibit a liquidcrystal-like nature and tend to form columnar phases, in which the nucleosomes are stacked one upon each other with their axes parallel to the column. A SH configuration exhibiting a columnar packing of nucleosomes in low-salt conditions is likely to be stabilized by internucleosomal interactions when the ionic force increases, better than any other configuration. In presence of linker histones, the specificity of "columnar" SH is strengthened since linker histones too exhibit a propensity to stacking.

\section{F. Comparison with chromatin fiber pulling experiments}

We carry on the discussion by presenting the experimental validation and prospects of our results.

In the past few years, a novel experimental method of investigating structural and mechanical properties of biological macromolecules or complexes, as DNA or chromosomes, has been developped [41] 11] [53] [54]. It is based on micromanipulations and force measurements on an isolated fiber. It belongs to the rapidly expanding field of investigations known as "single molecule biophysics" [42]. The experiment deviced in the context of chromatin study transposes to a chromatin fiber a methodology first implemented with DNA. It consists in pulling the fiber under various constraints and in various conditions (salt concentration, presence of specific enzymes or chemical factors), and to determine the ensuing deformations of the fiber. Varying the pulling force yields force-extension curves characterizing the elastic response of the fiber.

Nevertheless, at the chromatin scale, the applied stresses are artificial and cannot sensibly refer to an event occuring in vivo: contrary to DNA case, an enzyme is not large enough to directly handle the 30 -nm fiber or to experience the chromatin fiber state of strain, for example its torsional strain or its curvature (the only in vivo mechanism of that kind, i.e. the action of the mitotic spindle on chromosomes [8], occurs at a much larger scale). The biochemical processes, for instance binding of a biological factor or chemical modification, occur at the elementary level (scale of DNA and nucleosomes), whereas the experiment probes the 30-nm fiber behavior. A mechanical modeling relating the DNA scale and the fiber scale is thus necessary to exploit all the informations provided by single-fiber pulling experiments in terms of biological functions. Conversely, these experiments are essential to validate the model and the underlying hypotheses on structure and interactions, to fit dubious parameters and possibly to ask for refinements. The EWLR model allows to predict force-extension curves, to be compared with those obtained in single-fiber experiments. Our study, describing the microscopic root of the EWLR model of chromatin, thus provides a bridge between microscopic structure and observable properties of the overall fiber.

In the experimental setup used by Cui and Bustamante [6], the fiber, of relaxed length $L$, is pulled by means of optical tweezers. There is no direct contact with the fiber ends, which are free to rotate. No torque is applied $(\vec{M}=0)$ and free twist fluctuations take place. It corresponds to the situation recalled just above. Some of force-extension curves that they present are relaxation curves in low salt, for which it is legitimate to ignore interactions between nucleosomes. Cui and Bustamante fitted these experimental relaxation curves within the EWLR model presented in Section IV.A. Recalling that their experimental setup allows free twist fluctuations, the fit involved only two elastic constants: the bend persistence length $\mathcal{A}$ and an effective stretch modulus $\gamma_{\text {eff }}=\gamma-k_{B} T g^{2} / \mathcal{C}$. They found $\mathcal{A} \approx 30 \mathrm{~nm}$ and $\gamma_{\text {eff }} \approx 5 \mathrm{pN}$, values to which we may compare our theoretical predictions.

The comparison leads to a striking result: we evidence on Figure 11 that the set of values $\left(\mathcal{A} \approx 30 \mathrm{~nm}, \gamma_{\text {eff }} \approx 5\right.$ $\mathrm{pN}$ ) is obtained for $n_{b p}$ belonging to a narrow window between 42 and $43 \mathrm{bp}$ whatever the value of $\Phi$ is (we checked values of $\Phi$ ranging from $130^{\circ}$ to $40^{\circ}$ ). Since $n_{b p}$ is then an integral multiple of $n_{b p}^{0}$, this case corresponds to special structures of the $\mathrm{SH}$ in which the nucleosomes pack into columnar arrays (IV.E); only the number of columns varies with $\Phi$ and with the precise modeling of the chromatosome, see Figure 3. Pulling the fiber will induce a change in $\Phi$ but not in $\tau$, due to the special orientation of the nucleosomes; this orientation is thus preserved, hence $\gamma$ and $\mathcal{A}$ will not change: no nonlinear effect arises when starting in this special columnar geometry, hence a fit by an EWLR actually accounts for the whole force-extension curve, even in the region where $u$ is not small with respect to 1 .

In an experiment performed at higher salt concentration (in 40-150 mM NaCl) Cui and Bustamante observed a plateau in the force-extension curve at a value $F_{c}$ between $5 \mathrm{pN}$ and $6 \mathrm{pN}$. They interpret this plateau as a structural transition corresponding to the breaking of some short-range attractive interactions between chromatosomes, from which follows a dramatic decondensation of the fiber, at constant force. Our modeling cannot, of course, directly account for this conformational transition since internucleosomal interactions are ignored: only relaxation curves can be predicted, or curves in low salt conditions where interactions are always negligible, even at low force, and the chromatin always extended (there is besides no plateau in this low-salt case).

But we claim that our study is specially well-suited to investigate the relevant biological question, which is rather to determine local mechanisms that could in- 
duce such a decondensation, i.e. that could create local stresses of large enough strength to break the interactions. Indeed, a simple criterion of efficiency of biological factors (e.g. enzymes) is that the global stress resulting from their binding should be larger than the critical force $F_{c}$ measured by Cui and Bustamante. Our approach provides a direct method to check this criterion since it relates the local strains induced by the binding of the enzyme on linker DNA (experimentally measured or deduced from a molecular mechanics simulation) first to the associated local stresses (equation 11); then, by extending the computations presented in Section IV, it is possible to relate these local stresses to the global stress experienced by the chromatin fiber, to be compared to the measured value $F_{c}$, and to the global deformation of the fiber conformation. The implementation of this methodology in the case of intercalators, which gives insights on the in vivo condensation/decondensation mechanisms of the chromatin fiber, will be presented in a subsequent paper.

We mention that our work is not the first theoretical, model-based approach aiming at accounting for these experimental results.

A first one has been developped by Katritch et al. 44, using a Monte Carlo simulation of the fiber. One of the interests of such a simulation is to include explicitly thermal fluctuations but, as they show in their paper, the behavior experimentally observed is mainly deterministic (except at very low force). Moreover, the possibility offered by a simulation to treat accurately the interactions, for instance between nucleosomes, is at the moment hampred by the lack of data, preventing to go beyond an effective isotropic model of interaction. We thus believe that Monte Carlo simulations are not necessary, at least as concerns the problem of reproducing the experimentally observed force-extension curves. Indeed, an analytic answer is at hand, even with nucleosome interactions, and with more refined excluded-volume constraints (see Figure 7 and the analog that could be constructed for the linkers, by unwrapping the cylinder of radius $r$ ). Simulations would become really useful to handle a more detailed description of the chromatosome, for instance with an explicit linker histone.

A second approach has been performed recently by Schiessel et al. [7]. As discussed below (VII.B), its basic step is to relate the strains at the DNA and fiber scales (instead of the stresses, as performed here in Section IV). They only managed to determine some elastic constants (namely $\gamma$ and $\mathcal{A}$ ) of special geometries, and at the expense of some approximations, but they give analytical expressions directly in terms of the microscopic parameters ( here $l$ and $\Phi$ ). Moreover, the forces that they predict in their theoretical force-extension curves are smaller than the results of Cui and Bustamante by a noticeable factor of 4 . This could be explained by their derivation, summing up the bend and twist contributions to $\gamma^{-1}$, which is questionable. Indeed, looking at the ba- sic example of an helical spring shows that instead of writing $\gamma^{-1}(A, C)=\gamma^{-1}(A, C=0)+\gamma^{-1}(A=0, C)$, one should sum up the stretch moduli, according to $\gamma(A, C)=\gamma(A, C=0)+\gamma(A=0, C)$ as in the case when springs act in parallel. This leads to a discrepancy by a factor of 4 when the "elementary" strech modulus $\gamma(A, C=0)$ and $\gamma(A=0, C)$ are of the same order. Moreover, in the case of the $\mathrm{SH}$, the actual expression see equation (16) - shows that such an additive decomposition does not exactly hold in the $\mathrm{SH}$ case. These two objections could explain the discrepancy between our value of $5 \mathrm{pN}$ for $\gamma$, in agreement with the experimental results of Cui and Bustamante, and their value $\gamma=1.2$ $\mathrm{pN}$, yet observed for quite similar fiber conformations, with crossed linkers (Schiessel et al. considered a structure with (in our notations) $\Phi=85^{\circ}$ and $\tau=36^{\circ}$, which corresponds to $42-43$ bp in our model).

\section{BIOLOGICAL DISCUSSION}

\section{A. A tunable, highly sensitive, elastic structure}

We thus evidence a wide range of different elastic behaviors, separated by a minor change in the linker length. We suggest that this tunable elasticity might be used as a regulatory mechanism during the cell cycle. For instance, a slow modulation of the linker lengths, might create different domains in the chromatin fiber, of much different rigidities, and might provide a preliminary underlining of transcriptionally active chromatin regions. The response of these different regions to a same local stress (protein binding, for instance) will be dramatically different. For example, intercalation might condense or decondense the fiber, according to the sign of $\partial D / \partial \tau$, i.e. to the chirality: within $\Delta n_{b p}=2 \mathrm{bp}$, opposite consequences will be observed. This underlining, inscribed in the very structure of the fiber, allows a rapid and selected response to a non-specific stress, which might be biologically more relevant than a mechanism based on enzyme recognition of a specific sequence, moreover possibly buried inside the fiber. Mechanical sensitivity is likely to provide efficient switches for processes occuring at the fiber level.

The chromatin fiber thus exhibits tunable structure, tunable chirality and tunable elastic properties. We suggest three possible mechanisms to implement the required adaptation of the linker length:

- the first mechanism involves nucleosome displacement; acetylation of histone tails untightens the DNA wrapping around the histone core and presumably allows nucleosome mobility. Nevertheless, topological (linking number conservation) and mechanical (helical gearing) constraints make the motion of the nucleosome far different from a mere translation along the DNA, and the kinematic feasibility deserves to be investigated further; 
- the second mechanism involves intercalating enzymes, modifying the twist of the linker. A detailed study of the interplay between linker intercalation and the chromatin fiber mechanics will be presented in a subsequent paper;

- the best candidate might be a mechanism involving the linker histone. Indeed, the value $n_{b p}$ involved here is the effective length of the linker, beyond linker histone (i.e. outside the chromatosome). This length might be tuned by a slight displacement of the linker histone away $\left(\Delta n_{b p}<0\right)$ or towards $\left(\Delta n_{b p}>0\right)$ the nucleosome.

An alternative tuning mechanism lays on the variation of $\Phi$, controlled in particular by the presence of linker histone, salt concentration and histone tails binding affinities.

\section{B. A novel chromatin structure}

We evidenced that a value of linker length $n_{b p}$ between 42 and $43 \mathrm{bp}$ leads to the values $\mathcal{A} \approx 30 \mathrm{~nm}$ and $\gamma \approx 5$ $\mathrm{pN}$ whatever $\Phi$. Since $n_{b p}$ is then about $4 n_{b p}^{0}$, the nucleosome axes are all parallel hence parallel to the $\mathrm{SH}$ axis due to the rotational symmetry. That such organized structures lead to $\mathcal{A}=30 \mathrm{~nm}$ and $\gamma_{e f f}=5 \mathrm{pN}$, whatever $\Phi$ is (and even whatever $r_{n u c l}$ and $H$ are, as we checked) is explained by the fact that the $\mathrm{SH}$ elastic properties originate from the linker DNA contribution. As shown by the computation of SH elastic constants, this contribution is mainly fixed by the linker orientation with respect to the $\mathrm{SH}$ axis (i.e. $z$ ). This matching between our predictions and experimental results strongly suggests that the structure underlying the observed elastic properties is a columnar packing of nucleosomes. We thus supplement the argument of Yao et al. 45 in favour to rotationally phased nucleosomes: the actual relaxed $\mathrm{SH}$ structures are selected according to their ability to be stabilized by internucleosomal interactions when the ionic force increases. Our claim is supported by the results of Livolant et al. [39] 40, in which it is observed that nucleosomes exhibit a liquid-crystal-like nature, leading to a spontaneous columnar ordering. We thus expect native $\mathrm{SH}$ structures to favour columnar packing of nucleosomes, as they correspond to the more robust three-dimensional organization of the chromatin fiber. Note that whereas a model with straight linkers is compatible with the observed structure and elasticity of the chromatin fiber at low ionic strength $(5 \mathrm{mM} \mathrm{NaCl})$, interactions between stacked nucleosomes should induce a bending of the linkers at higher ionic strength. More probably, the conflicting effects consistency between nucleosomes stacking and linker stiffness might be reconciled by linker DNA kinks, occuring near the entry/exit points and induced by the binding oh linker histones $\mathrm{H} 1$.

For $\Phi=90^{\circ}$ (low-salt situation), the degree of compaction $10 / D(\mathrm{~nm})$ reaches its minimal value for the same value $n_{b p}$ located between 42 and $43 \mathrm{bp}$. This under- lines a key feature of the corresponding configuration: at low salt, it is the most extended and rigid configuration; at the same time, it is the most responsive to salt induced compaction. Indeed, as seen on Figure 9, the degree of compaction $10 / D(\mathrm{~nm})$ is minimal at $n_{b p}=42$ or 43 bp for $\Phi=90^{\circ}$, and it strongly increases when $\Phi$ decreases to $50^{\circ}$, which is an acknowledged effect of increasing the salt concentration. Moreover, this configuration strongly favours a second compaction step, ensured by the attractive interactions between nucleosomes (or rather chromatosomes) that arise when the nuclesome faces are close enough. We in particular recover in this scheme the two-stage compaction of the 30-nm chromatin fiber observed experimentally [46] [47]. An insight on this interaction-induced compaction can be obtained by setting the effective parameters $r_{n u c l}$ and $H$ to 0 , thus mimicking the enhanced influence of linker histone at high salt; in this case, $P$ decreases to about $6 \mathrm{~nm}$, indicating that nucleosomes actually stack very closely onto each other and lead to a superstable (and presumably rigid) fiber. It has been suggested [31] that H1 is required not so much to get a folded fiber (compact fibers have been observed in absence of $\mathrm{H} 1$ ) but to get a properly folded fiber. We suggest that $\mathrm{H} 1$ might be involved in the tuning of the effective linker length and twist, actually involved in the assembly. In any cases, H1 stacking interactions favour configurations exhibiting columnar arrays of nucleosomes.

\section{CONCLUSION AND PERSPECTIVES}

\section{A. What did we learn from this study?}

The chromatin sensitivity of the fiber response to global or local stresses evidenced in this study sheds light on the biological interest of the so peculiar and so universal assembly of chromatin fiber. It enlights possible relations between small-scale structure and gene regulation through the fiber mechanical properties. For instance, we evidenced that a minor change of $l$ or $\tau$ around some "critical" value inverts its chirality. Hence, its response to a torsional torque induces either an extension, either a contraction of the fiber, and its response to a pulling force will either winds or unwinds the $\mathrm{SH}$ fiber. Moreover, as DNA itself is chiral (right-handed), the response at the DNA level is also controlled by the SH chirality: pulling the fiber unwinds the DNA if the $\mathrm{SH}$ is left-handed.

The tunable energy partition between twist and bend degrees of freedom at the linker DNA level may be of biological interest: according to the chromatin configuration, either a twist-sensitive protein will bind onto linker DNA, either a protein whose binding is favoured by the local curvature of linker DNA will be the adapted factor. Hence the mechanical sensitivity can participate to biological recognition or specificity. Conversely, we read on the associated curves (see Figure 11) whether 
a twist-modifying protein (intercalator, gyrase), for instance, may induce a required strain of the $\mathrm{SH}$.

Comparison with experimental results give clues about the much debated chromatin structure [36]. It brings about a novel structure: a columnar packing of nucleosomes. We suggest that a possible role of linker histone might be to select the proper structure, at low salt, by tuning the linker length so as to have $n_{b p} / n_{b p}^{0}$ equal to some integer. Also, a tuning of $\Delta n_{b p}=1$ or 2 bp might be achieved by enzyme intercalation. When ionic strength increases, compaction takes place, first due to a decrease of $\Phi$ (which keeps the nucleosome orientations unchanged, along the SH axis); then, when nucleosomes happen to be stacked in columns, the compaction is ensured by the interactions between stacked nucleosomes, between stacked linker histones (counterbalancing the linker DNA repulsion) or between histone tails and nearby linkers or nucleosomes.

\section{B. A general method for studying elasticity of linear complex fibers}

The problem of relating the elastic coefficients of the chromatin fiber to the geometric and elastic properties of the underlying "microscopic" structure (assembly of nucleosomes and linkers) is reminiscent of similar works performed for DNA by Marko and Siggia [52] and O'Hern et al. [17] 22]. They described the dsDNA at two levels: as an helical coiling at small scale and as an EWLR at a slightly larger scale. They derived similar formulas relating the elastic coefficients $\left(C, A, \gamma_{D N A}\right.$ and $\left.g_{D N A}\right)$ to the geometric and elastic parameters of the underlying helical model.

We point that their computation rests on the relation between the "microscopic" strains and the dsDNA strains, plugged into the equality of the EWLR free energy and the free energy computed within the microscopic model. Generally, numerous sets of microscopic strains achieve the macroscopic strains, but only the set of lowest free energy yields the actual free energy of the EWLR. Relating properly microscopic and macroscopic strains thus requires to minimize the small-scale free energy, given the macroscopic strains. Performing this minimzation is nothing but writing the conditions for the local equilibrium of the assembly.

When the microscopic model is homogeneous, the conditions for local equilibrium simply expresses in the extensivity of the strains. The uniformity of the local strain densities thus allows to relate them to global strains without an explicit minimization (think to identical springs in series). This works for DNA [22] but not in the case of chromatin. The discrete and complex nature of the chromatin assembly leads to difficult and cumbersome computations in order to determine the linker shape, as it can be seen in the work of Schiessel et al. [7]. Moreover, this approach fails to give an analytical solution except when the relaxed fiber exhibits a special geometry, for example a ribbon-like flat structure.

Determining the conditions for local equilibrium is precisely what is done, more straighforwardly, in our approach. We indeed write equilibrium equations given the global stresses and solve them to get the local stresses arising in each point of the assembly (at equilibrium under the given global stresses). Relating the stresses at the microscopic and at the fiber levels follows from the basic principles of classical mechanics; this method appears to be at the same time more simple and more easily generalized. It is in fact the only way to bridge the linker elasticity to the SH elasticity in any geometry. Moreover, it extends to more complex situations as intercalated linker DNA or more generally, situations where forces and torques are applied at the DNA level.

We thus underline that the proper method to express the elastic properties of an assembly as a function of the elastic properties of the basic elements is to relate the global stresses, applied to the assembly, and the local stresses experienced by the lower scale elements. This relation can be used in both ways, to investigate

- either small-scale repercussions of a global stress, hence how a global stress (as those applied in micromanipulations) can be used to probe the fiber at the elementary level,

- either large-scale response to local stresses, hence how biological factors binding on the linker DNA could induce major structural and conformational changes in the overall fiber.

\section{Biological perspectives}

Our study underlines that the mechanical properties of special structures, selected according to the phasing of the nucleosomes therein, might be involved as a regulatory factor in the chromatin biological function.

Having modelled the 30-nm fiber as a EWLR, with explicit values of the elastic coefficients, a natural extension of our study is to consider higher levels of organization: a plectonemic coiling, leading to a $60-\mathrm{nm}$ fiber, or an helical coiling, whose elastic properties follow from classical spring mechanics. The question is then to unravel the implications of the chromatin structure and its elastic properties on the higher levels of organization. A mechanical approach similar to that implemented in this paper is essential to bridge electron-microscopy structural observations evidencing fiber-like objects at higher levels (60-nm fiber, "chromonema" fiber of diameter 100-130 nm 55 and chromosome) and experimental results on chromosome elasticity obtained by pulling a single chromosome [53 54. The challenge is to understand the mechanics of the chromosome and its involvment in the biological functioning of the chromosome throughout the cell cycle.

Another direction in which to exploit the results of the present paper is to determine the stresses that can be 
exerted at the fiber scale by groove-binding proteins or intercalators when they bind onto linker DNA. For instance investigate whether a local decondensation of the fiber might be induced by intercalating enzymes and controlled by linker lengths. More generally, our approach is a privileged tool to investigate the action at the fiber level of small-scale biochemical stresses (protein binding, histone tail acetylation), then to describe how they can act as mechanical switches and exploit the tunable elasticity of the fiber into regulatory schemes.
[1] K. van Holde, Chromatin, Springer, Berlin (1988).

[2] A. Wolffe, Chromatin, Academic Press, San Diego (1995).

[3] W. Saenger, Principles of nucleic acid structure, ch. 19, Springer, Berlin (1988).

[4] J. Widom, Chromosome structure and gene regulation Phys. A 244, 497 (1997).

[5] A.E.H. Love, A treatise on the mathematical theory of elasticity, 4th edition, chapters XVIII and XIX, Cambridge, London (1986).

[6] Y. Cui and C. Bustamante, Pulling a single chromatin fiber reveals the forces that maintain its higher-order structure, Proc. Natl. Acad. Sci. USA, 97, 127 (2000).

[7] H. Schiessel, W.M. Gelbart, and R. Bruinsma, Structural and mechanical properties of the two-angle model for chromatin, Biophys. J. 80, 1940-1956 (2001).

[8] G. Janninck, B. Duplantier and J.L. Sikorav, Forces on chromosomal DNA during anaphase, Biophys. J. 71451 (1996).

[9] J. Widom, Structure, dynamics and function of chromatin in vitro, Annu. Rev. Biophys. Biomol. Struc. 27, 285 (1998).

[10] C.L. Woodcock, S.A. Grigoryev, R.A. Horowitz and N. Whitaker, A chromatin coiling model that incorporates linker variability generates fibers resembling the native structures, Proc. Natl. Acad. Sci. USA 90, 9021 (1993).

[11] T.R. Strick, J.F. Allemand, D. Bensimon, A. Bensimon and V. Croquette, The elasticity of a single supercoiled DNA molecule, Science 271, 1835 (1996).

[12] C. Bouchiat and M. Mézard, Elasticity model of a supercoiled DNA molecule, Phys. Rev. Lett. 80, 1556 (1998).

[13] K. Luger, A.W. Mäder, R.K. Richmond, D.F. Sargent and T.J. Richmond, Crystal structure of the nucleosome core particle at 2.8 A resolution, Nature 389, 251 (1997).

[14] O. Kratky and G. Porod, Rec. Trav. Chim. 68, 1106 (1949)

[15] J. Marko, Stretching must twist DNA, Europhys. Lett. 38, 183 (1997).

[16] J. Marko, DNA under tension: overstretching, undertwisting and relaxation dynamics, Phys. Rev. E 57, 2134 (1998).

[17] R.D. Kamien, T.C. Lubensky, P. Nelson and C.S. O'Hern, Twist-stretch elasticity of DNA, Europhys. Lett. 38, 237 (1997).

[18] P.T. Lowary and J. Widom, Nucleosome packaging and nucleosome positioning of genomic DNA, Proc. Natl. Sci. Acad. USA 94, 1183 (1997).

[19] J. Bednar, R.A. Horowitz, S.A. Grigoryev, L.M. Carruthers, J.C. Hansen, A.J. Koster and C.L. Woodcock, Nucleosomes, linker DNA, and linker histone form a 
unique structural motif that directs the higher-order folding and compaction of chromatin, Proc. Natl. Acad. Sci. USA 95, 14173 (1998).

[20] L.M. Carruthers, C. Tse, K.P. Walker III and J.C. Hansen, Assembly of defined nucleosomal and chromatin arrays from pure components, in Chromatin, Meth. in Enzym. 304, edited by P. Wassarman and A.P. Wolffe, p.19 (1999).

[21] K. van Holde and J. Zlatanova, What determines the coiling of the chromatin fiber? Proc. Natl. ad. Sci. USA 93, 10548 (1996).

[22] C.S. O'Hern, R.D. Kamien, T.C. Lubensky and P. Nelson, Elasticity theory of a twisted stack of plates, Eur. Phys. J. B1, 95 (1998).

[23] Z. Kam, N. Borochov and H. Eisenberg, Dependence of laser light scattering of DNA on $\mathrm{NaCl}$ concentration, Biopolymers 20, 2671 (1981).

[24] T. Odijk, J. Polym. Sci. 15, 477 (1977).

[25] R.R. Netz and H. Orland, Variational theory for a single polyelectrolyte chain, Eur. Phys. J. B 8, 81 (1999).

[26] C. Baumann, S. Smith, V. Bloomfield and C. Bustamante, Ionic effects on the elasticity of single DNA molecules, Proc. Natl.Acad. Sci. USA 94, 6195 (1997).

[27] S.H. Leuba, C. Bustamante, J. Zlatanova and K. van Holde, Contributions of linker histones and histone H3 to chromatin structure: scanning force microscopy studies on trypsinized fibers, Biophys. J. 74, 2823 (1998).

[28] S.H. Leuba, G. Yang, C. Robert, B. Samori, K. Van Holde, J. Zlatanove and C. Bustamante, Threedimensional structure of extended chromatin fibers as revealed by tapping-mode scanning force microscopy, Proc. Natl. Acad. Sci. USA 91, 11621 (1994).

[29] J.O. Thomas, Histone H1: location and role, Curr. Opin. Cell Biol. 11312 (1999).

[30] L.M. Carruthers, J. Bednar, C.L. Woodcok and J.C. Hansen, Linker histones stabilize the intrinsic saltdependent folding of nucleosomal arrays: mechanistic ramifications for higher-order chromatin folding, Biochemistry 37, 14776 (1998).

[31] J. Zlatanova, S.H. Leuba and K. van Holde, Chromatin structure revisited, Crit. Rev. Eukaryot. Gene Expr. 9, 245 (1999).

[32] S.H. Leuba, C. Bustamante, K. van Holde and J. Zlatanova, Linker histones tails and $\mathrm{N}$-tails of histone $\mathrm{H3}$ are redundant: scanning force microscopy studies of reconstituted fibers, Biophys. J. 74, 2830 (1998).

[33] A. Worcel, S. Strogatz and D. Riley, Structure of chromatin and the linking number of DNA, Proc. Natl. Acad. Sci. 78, 1461 (1981).

[34] D.Z. Staynov, Possible nucleosome arrangements in the higher-order structure of chromatin, Int. J. Macromol. 5, 3 (1983).

[35] J.T. Finch and A. Klug, Proc. Natl. Acad. Sci. USA 73, 1897 (1976).

[36] K. van Holde and J. Zlatanova, Chromatin higher order structure: chasing a mirage?, J. Biol. Chem. 270, 8373 (1995).

[37] S. Przybyl and P. Pieranski, Helical close packing of ideal ropes, to appear in Eur. Phys. J. E (2001).

[38] J.W. Phillips and G.A. Costello, General axial response of stranded wire helical springs, Int. J. Non-Linear Mechanics, 14247 (1979).

[39] A. Leforestier and F. Livolant, Liquid crystalline ordering of nucleosome core particles under macromolecular crowding conditions: evidence fora discotic columnar hexagonal phase, Biophysical J. 73, 1771 (1997).

[40] F. Livolant and A. Leforestier, Chiral discotic columnar germs of nuclosome core particles, Biophysical J. 78, 2716 (2000).

[41] S.B. Smith, Y. Cui and C. Bustamante, Overstretching $B-D N A$ : the elastic response of individual double-stranded and single-stranded DNA molecules, Science 271, 795 (1996).

[42] Science 283, special issue on single molecules (1999).

[43] R.R. Netz, Strongly stretched semiflexible extensible polyelectrolytes and DNA, preprint (2001).

[44] V. Katritch, C. Bustamante and W.K. Olson, Pulling chromatin fibers: computer simulations of direct physical micromanipulations, J. Mol. Biol. 295, 29 (2000).

[45] J. Yao, P.T. Lowary and J. Widom, Twist constraints on linker DNA in the 30-nm chromatin fiber: implications for nucleosome phasing, Proc. Natl. Acad. Sci. 90, 9364 (1993).

[46] I. Russo, P. Barboro, I. Alberti, S. Parodi and C. Balbi, Role of $H 1$ in chromatin folding. A thermodynamic study of chromatin reconstitution by differential scanning calorimetry, Biochemistry 34, 301 (1995).

[47] C. Balbi, P. Sanna, P. Barboro, I. Alberti, M. Barbesino and E. Patrone, Chromatin condensation is confibed to the loop and involves an all-or-non structural change, Biophys. J. 77, 2725 (1999).

[48] J.H. White, Am. J. Math. 91, 693 (1969).

[49] F.H.C. Crick, Linking numbers and nucleosomes, Proc. Natl. Acad. Sci. USA 73, 2639 (1976).

[50] B. Fain, J. Rudnick and S. Östlund, Conformations of linear DNA, Phys. Rev. E 55, 7364 (1997).

[51] P. Nelson, New measurements of DNA twist elasticity, Biophys. J. 74, 2501 (1998).

[52] J. Marko and E.D. Siggia, Bending and twisting elasticity of DNA, Macromol. 27, 981 (1994).

[53] B. Houchmandzadeh, J.F. Marko, D. Chatenay and A. Libchaber, Elasticity and structure of eukaryote chromosomes studied by micromanipulation and micropipette aspiration, J. Cell Biol. 139, 1(1997).

[54] M. Poirier, S. Eroglu, D. Chatenay and J.F. Marko, reversible and irreversible uncoiling of mitotic newt chromosomes by applied force, Mol. Biol. Cell 11, 269 (2000).

[55] A.S. Belmont, S. Dietzel, A.C. Nye, Y.G. Strukov and T. Tumbar, Large-scale chromatin structure and function Curr. Opin. Cell Biol. 11, 307 (1999). 


\section{CAPTIONS}

Figure 1: Geometric description of linker DNA (section II.A). Above: the arrows indicate the orientation of the two strands, delimiting the minor groove (filled in grey) and the major groove. The vector $\vec{t}(s)$ is tangent to the minor groove and rotates around the local axis $\vec{u}$ (straight in a relaxed linker) with an angular rate $\omega^{0}=2 \pi / l^{0}$; the two vectors make a constant angle $90^{\circ}-\alpha_{D N A} \approx$ $62^{\circ}$. Below: tranverse view; the bold contour locates the minor groove.

Figure 2: Nucleosome modeling and assembly of the chromatin fiber (section II.B). Left above: view in perspective of the nucleosome $j ; \alpha$ is the slope of the DNA left-handed wrapping around the histone core $(\alpha \approx$ $4.47^{\circ}$ ). The dyad axis $\vec{D}_{j}$ is orthogonal to the nucleosome axis $\vec{N}_{j}$. Left below: view from above (projection on a plane orthogonal to the nucleosome axis. Right: vectors $\vec{N}_{j}, \vec{u}_{j}$ and $\vec{t}_{j}(l)$ in the plane tangent to the nucleosome at $E_{j}$.

Figure 3a: Various SH structures obtained when the parameter $l$ (equivalently $n_{b p}$ ) is varied, for $\Phi=90^{\circ}$. Note the columnar packing $(\beta \approx 0)$ obtained for $n_{b p}$ between $42 \mathrm{bp}$ and $43 \mathrm{bp}$, and the ribbon-like structure (two nucleosomes per turn, $\theta=\pi$ ) obtained for $n_{b p}=47 \mathrm{bp}$, whatever the value of $\Phi$. In between, the fiber exhibits cross-linked configurations. In each period of length 10.6 $\mathrm{bp}$, an interval (depending of $\Phi$ ) of $n_{b p}$-values is forbidden as it corresponds to self-overlapping configurations (here around $n_{b p}=38 \mathrm{bp}$ )

Figure 3b: Various SH structures obtained when the parameter $l$ (equivalently $n_{b p}$ ) is varied, for $\Phi=50^{\circ}$. Configurations around $n_{b p}=45 \mathrm{bp}$ are forbidden (steric hindrance).

Figure 4: Left: $\mathrm{SH}$ from above (projection in a plane orthogonal to the SH axis $\vec{A}$, here for $\beta=90^{\circ}$. We define $R$ as the distance from any nucleosome center $G_{j}$ to the $\mathrm{SH}$ axis (measured along the dyad axis $\vec{D}_{j}$ ), $r$ as the distance of any entry (or exit) point to the SH axis $\vec{A}$; the "excluded-volume" radius $R_{S H}$ of the $\mathrm{SH}$, i.e. the radius of the cylinder of axis $\vec{A}$ containing the whole fiber (including the nucleosomes) satisfies $R_{S H}=R+$ $r_{\text {nucl }}$ due to the peculiar orientation of the nucleosomes (the axes $\vec{N}_{j}$ are tangent to the cylinder of axis $\vec{A}$ and radius $R$ whatever $\beta$ ). We also introduce the angle $\theta$ of the rotation around the SH axis $\vec{A}$, transforming the projection of a nucleosome into the following one, and the angle $\eta$ between the projections of the vectors relating $S_{j-1}$ and $E_{j}$ to the $\mathrm{SH}$ axis. This angle $\eta$ is chosen in $[0,2 \pi[$. It satisfies $l \sin z=2 r \sin (\eta / 2)$. Right: projection of the first nucleosome in the plane orthogonal to its axis
$\vec{N}_{1}$. Note that the nucleosome dyad axis is orthogonal both to the nucleosome axis (nucleosome symmetry) and to the $\mathrm{SH}$ axis (symmetry of the assembly).

Figure 5: Left: Location of the nucleosome axis $\vec{N}_{j}$ in the frame spanned by the SH axis $\vec{A}$ and the nucleosome $j$ dyad axis $\vec{D}_{j}$; it lays in the plane orthogonal to $\vec{D}_{j}$ and makes an angle $\beta \in[0,2 \pi]$ with respect to $\vec{A}$. This angle, varying with $\Phi$ and $l$, satisfies $\cos \beta=\vec{N}_{j} \cdot \vec{A}$. Right: Location of the linker $\vec{u}_{j}$ in the frame spanned by the SH axis $\vec{A}$ and the nucleosome dyad axis $\vec{D}_{j}$; the linker makes a constant angle $\xi$ with the dyad axis.

Figure 6: Front view of the $\mathrm{SH}$ projected in a plane spanned by the SH axis $\vec{A}$ and the linker axis $\vec{u}_{j}$. The $\mathrm{SH}$ axis is actually ahead of the linker, which lays in the figure plane; $E_{j-1}, G_{j-1}, S_{j}$ and $G_{j}$ are projections on the figure plane. Only the track of the nucleosomes is indicated: neither their axes nor their dyad axes belong to the drawing plane. $D$ is the distance between two successive nucleosomes along the $\mathrm{SH}$ axis $\vec{A}$, whose orientation is chosen so that $D>0$. We denote $z$ the angle between the linker and the SH axis; it satisfies $\vec{u}_{j} \cdot \vec{A}=\cos z$ and by convention $z \in[0, \pi[$ hence $\sin z>0$.

Figure 7: Unwrapping of the cylinder of radius $R$ (containing the nucleosome centers). The construction only requires to know the values of $\beta, \theta, D$ and the nucleosome dimensions $r_{n u c l}$ and $H$. This drawing can be conveniently used to check the excluded-volume constraint between any two nucleosomes. Linkers, crossing the plane of the drawing, are not represented, nor the entry and exit point (located outside the drawing plane).

Figure 8: Fiber actual diameter $2 R_{S H}$ (dashed upper line, curve (1)) and pitch $P$ (solid line, curve (2)) in $\mathrm{nm}$, versus linker length (in bp), for $\Phi=90^{\circ}$ (left) and $\Phi=50^{\circ}$ (right). As what matters is the twist angle $\tau=2 \pi n_{b p} / n_{b p}^{0}$, the number $n_{b p}$ of bp is allowed to vary continuously. The thin line (3) represents the distance $D$ between two nucleosomes along the $\mathrm{SH}$ axis. The line made of crosses (4) represents the lower bound $P_{c}$ on the pitch (equation 3). The configurations with $P>P_{c}$ satisfy excluded-volume constraints.

Figure 9: (Solid line) compaction rate 10/D(nm) (number of nucleosomes per $10 \mathrm{~nm}$ of fiber) and (crosses) number $2 \pi / \theta$ of nucleosomes per turn versus linker length (in bp), for $\Phi=90^{\circ}$ (left) and $\Phi=50^{\circ}$ (right). Two nucleosomes per turn correspond to ribbon-like configurations, whereas the maximal number is reached for columnar packing $\left(n_{b p}=43 \mathrm{bp}\right)$.

Figure 10: $\operatorname{Cos}(\eta / 2)$ (solid line, (1)), $\cos \beta$ (dashed line (2)) and $\cos z$ (crosses (3)) versus linker length (in bp), 
for $\Phi=90^{\circ}$ (left) and $\Phi=50^{\circ}$ (right). $\operatorname{Cos}(\eta / 2)=0$ corresponds to a change in the $\mathrm{SH}$ chirality; $\cos \beta=1$ corresponds to columnar packing in which the nucleosomes axes are parallel to the $\mathrm{SH}$ axis: $\cos z$ is then small and the linkers are almost orthogonal to the axis (the difference is $\alpha \approx 4.5^{\circ}$ where $\tan \alpha$ is the slope of the linkers on the nucleosomes).

Figure 11: Above: Twist persistence length $\mathcal{C}$ (dashed line, curve (1)), bend persistence length $\mathcal{A}$ (solid line, curve (2)) and SH pitch $P$ (lower thick line, curve (3)) in $\mathrm{nm}$ versus linker length (in bp, according to $l=$ $l^{0} n_{b p} / n_{b p}^{0}$ ) for $\Phi=90^{\circ}$ (left) and $\Phi=50^{\circ}$ (right). Note that they all vanish at a critical value $n_{b p, c}$, strongly depending on $\Phi$ and on the detailed modeling of the nucleosome (or chromatosome). The dotted line (curve (4)) represents the lower bound $P_{c}$ (equation 3) indicating the values of $n_{b p}$ forbidden by steric hindrance. Middle stretch modulus $\gamma$ (solid line, curve (1)) and effective stretch modulus $\gamma_{e f f}=\gamma-k_{B} T g^{2} / \mathcal{C}$ (dashed line, curve (2)) in $\mathrm{pN}$ for $\Phi=90^{\circ}$ (left) and $\Phi=50^{\circ}$ (right). $B e$ low: twist-stretch coupling $g$ (no dimension) versus linker length (in bp) for $\Phi=90^{\circ}$ (left) and $\Phi=50^{\circ}$ (right).

Figure 12: these figures represent the relative contribution (in \%) of bending energy (curves (1)) and twist energy (curves (2)) to the total elastic energy stored in a linker (within the chromatin superhelix), versus linker length (in bp), in various instances: (above) when a force $\vec{F}=F \vec{A}$ is applied along the $\mathrm{SH}$ axis $\vec{A}$ but without any torque, (middle) when a torsional torque $\vec{M}_{t}=M_{t} \vec{A}$ is applied but no force, (below) when a flexural torque ( $\left.\vec{M}_{b} \cdot \vec{A}=0\right)$ is applied but no force. Two values of the angle $\Phi$ have been investigated: $\Phi=90^{\circ}$ (left) or $\Phi=50^{\circ}$ (right). The almost flat line, below $2 \%$, in the two upper figures, corresponds to the fraction of energy corresponding to stretch deformations; it is obviously negligible.

We compare these curves with those obtained for an helical coiling of DNA (section V.B) having the same values of $z$ and $r$ (crosses, curve (3) for the twist energy and diamonds, curve (4) for the bending energy). When applying only a pulling force, the partitions strongly differ; by contrast, the partitions observed when only a torque (either $M_{t}$, either $\vec{M}_{b}$ ) is applied are exactly identical (the curves superimpose).

Figure 13: Twist persistence length $\mathcal{C}$ (dashed line, curve (1)), bend persistence length $\mathcal{A}$ (solid line, curve (2)) in nm of an helical spring versus linker length (in bp) with the values of $z$ and $r$ equal to those of the $\mathrm{SH}$, respectively for $\Phi=90^{\circ}$ (left) and $\Phi=50^{\circ}$ (right). This shows that the decrease of the persistence lengths and their vanishing in special values $n_{b p, c}$ is a geometric effect: it is due to an accumulation of turns on a small distance along the axis, allowing a dramatic unfolding.
Figure 14: Ribbon-like structure of the $\mathrm{SH}(\theta=\pi)$, here projected in the plane $(\vec{D}, \vec{N} \wedge \vec{D})$; indeed, when $\theta=\pi$, all the planes $\left(\vec{D}_{j}, \vec{N}_{j} \wedge \vec{D}_{j}\right)$ coincide. The $\mathrm{SH}$ axis makes an angle $(\pi / 2-\beta)$ quite small with this plane $(\pi / 2-\beta=$ $\left.\alpha \approx 4.47^{\circ}\right)$ the nucleosomes are almost orthogonal to $\vec{A}$. Obviously, $D \sin \beta=l \sin (\Phi / 2)$. 


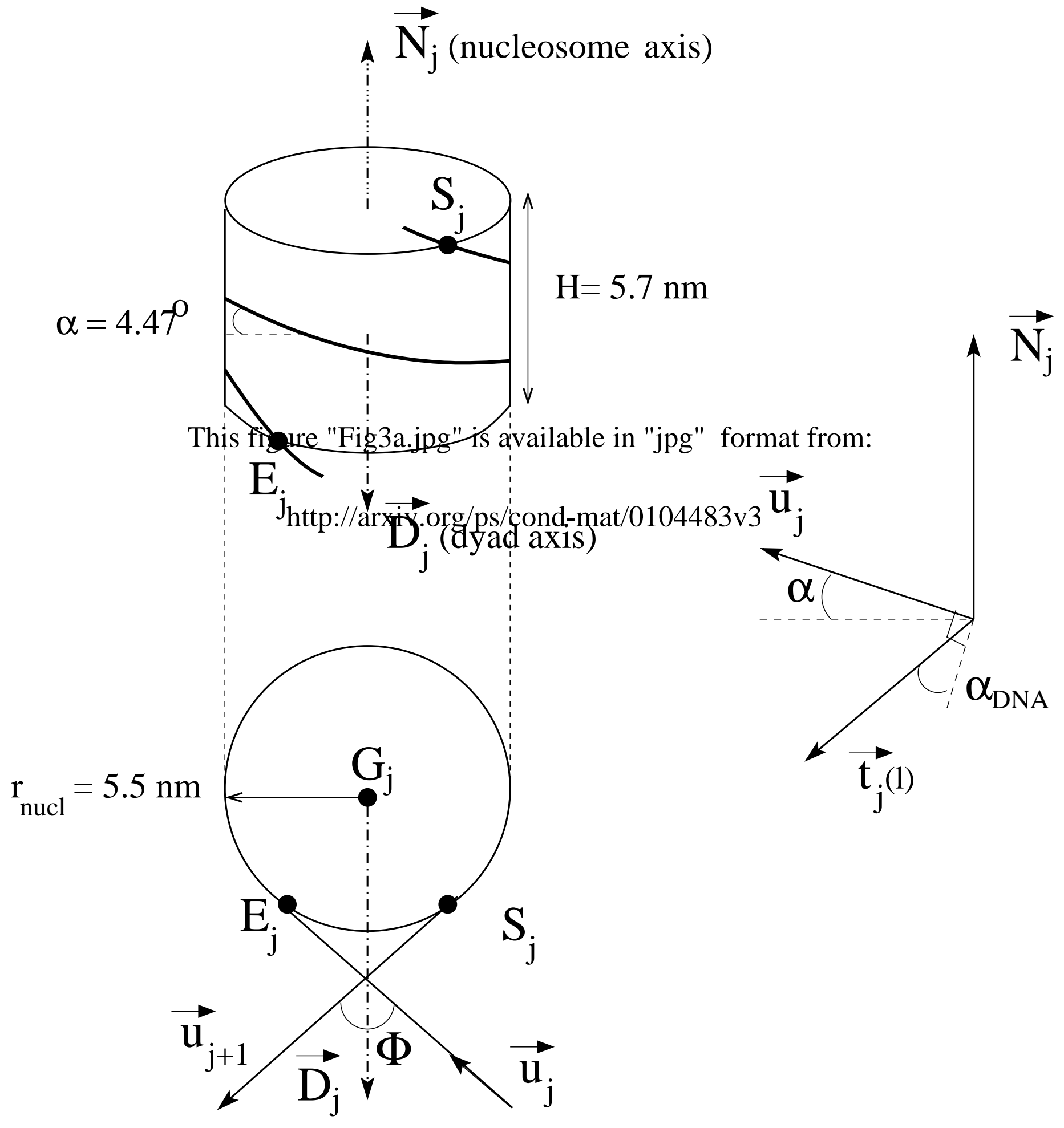


This figure "Fig3b.jpg" is available in "jpg" format from: http://arxiv.org/ps/cond-mat/0104483v3 


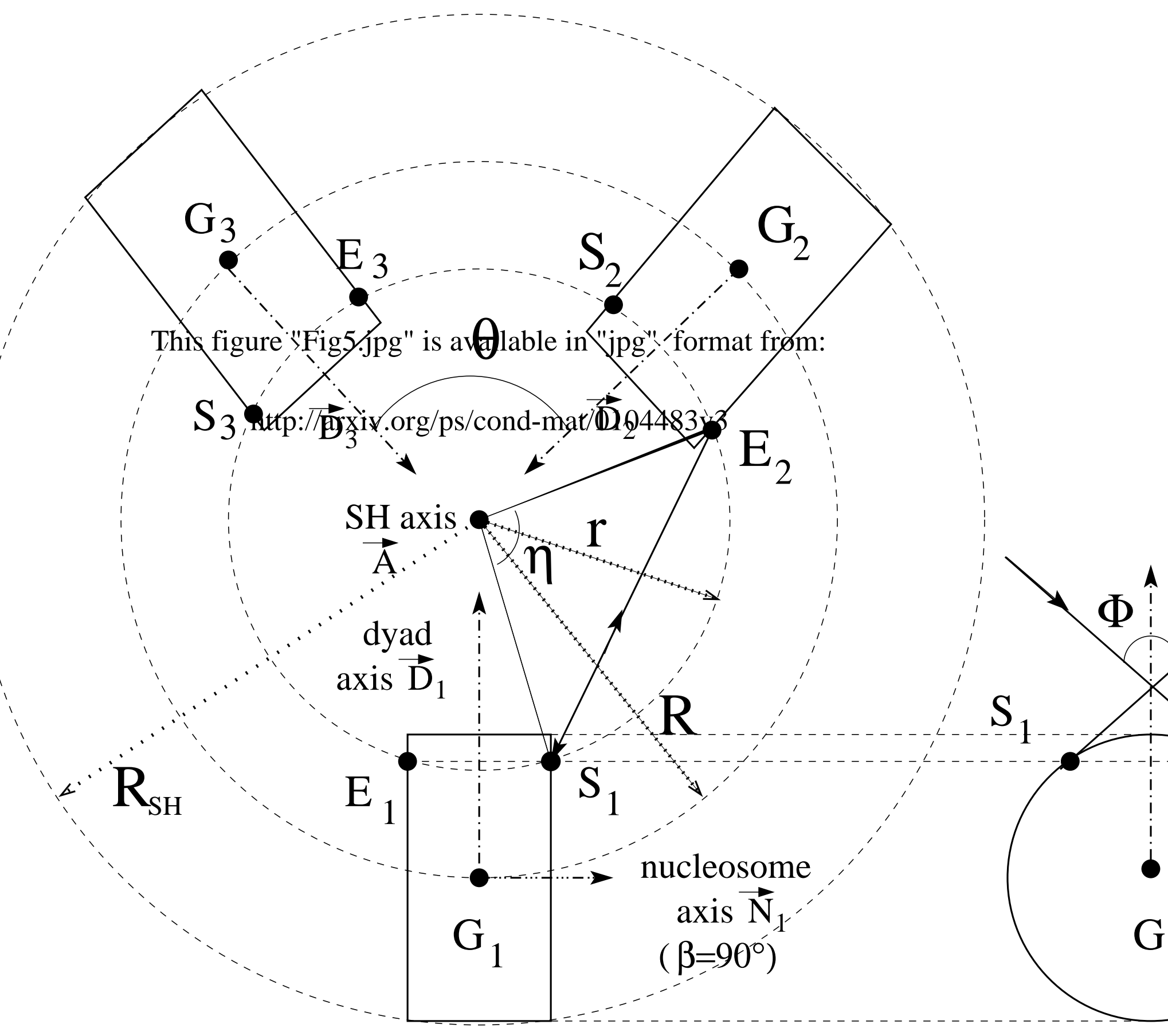



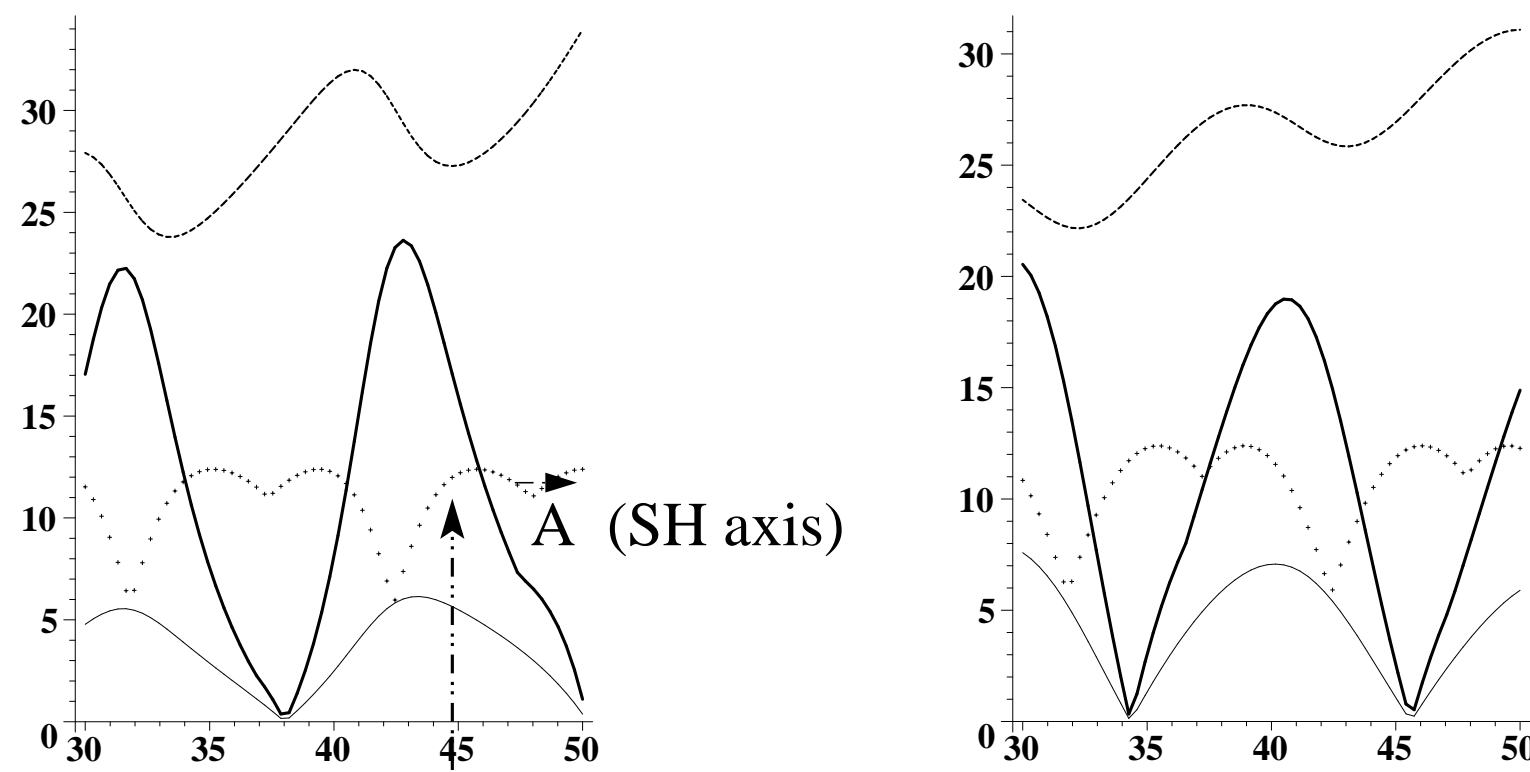

$E_{i}=P_{i}(l)$

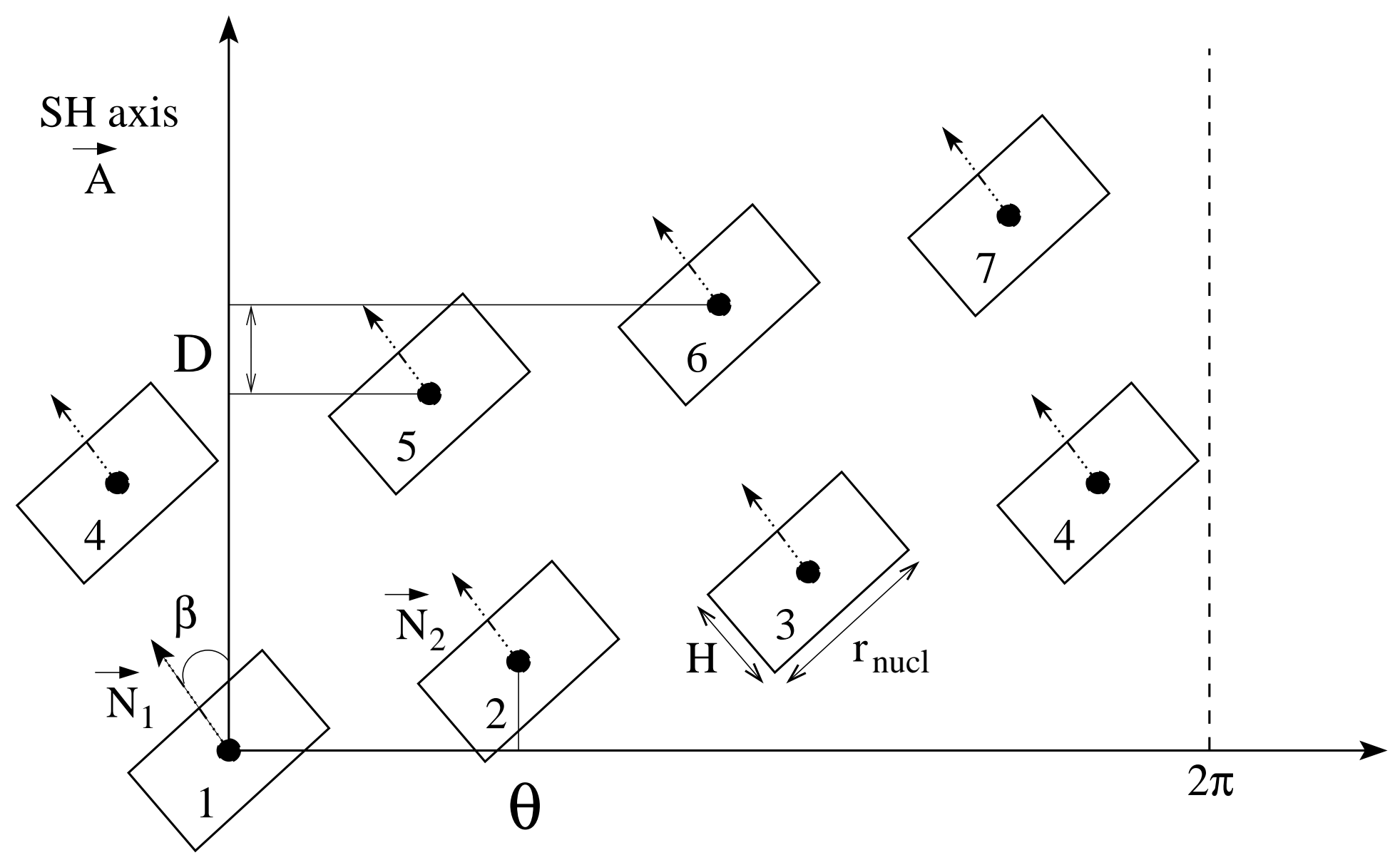


This figure "Fig9.jpg" is available in "jpg" format from: http://arxiv.org/ps/cond-mat/0104483v3 

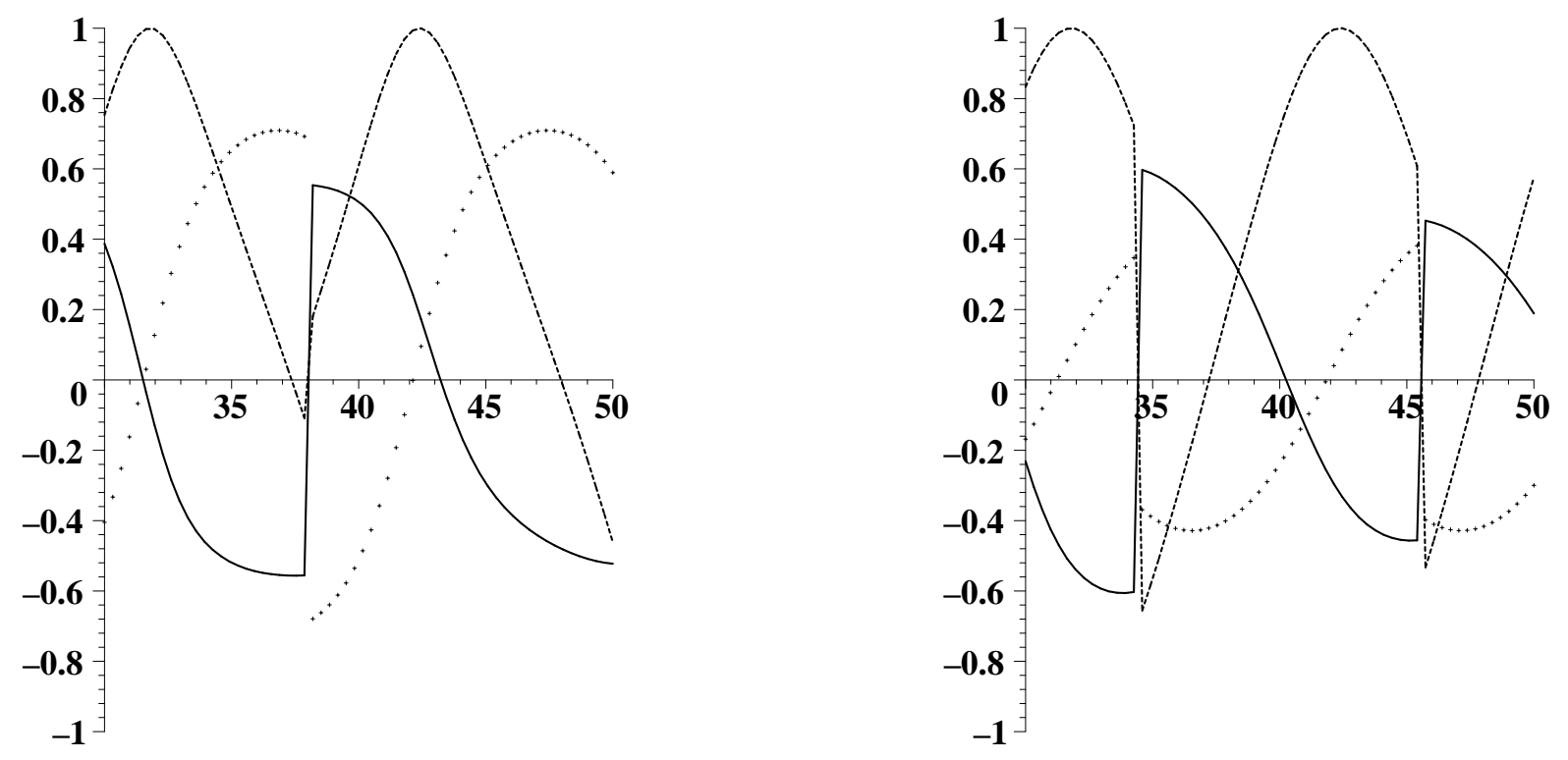
This figure "Fig11.jpg" is available in "jpg" format from: http://arxiv.org/ps/cond-mat/0104483v3 
This figure "Fig12.jpg" is available in "jpg" format from: http://arxiv.org/ps/cond-mat/0104483v3 

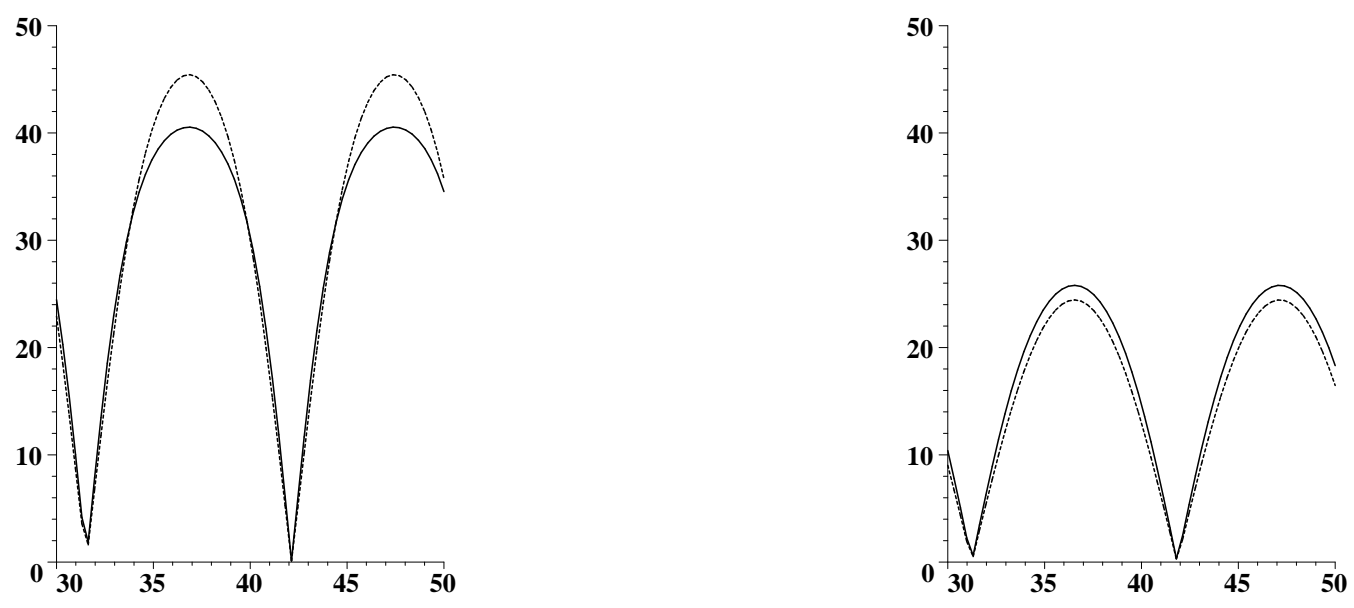